\title{
Joint Direction-of-Departure and Direction-of-Arrival Estimation in a UWB MIMO Radar Detecting Targets with Fluctuating Radar Cross Sections
}

\author{
Idnin Pasya, ${ }^{1,2}$ Naohiko Iwakiri, ${ }^{3}$ and Takehiko Kobayashi ${ }^{1}$ \\ ${ }^{1}$ Wireless Systems Laboratory, Tokyo Denki University, 5 Senju-Asahi-Cho, Tokyo 120-8551, Japan \\ ${ }^{2}$ Faculty of Electrical Engineering, Universiti Teknologi MARA, 40450 Shah Alam, Selangor, Malaysia \\ ${ }^{3}$ The University of Tokyo, 7-3-1 Hongo, Bunkyo-ku, Tokyo 113-0033, Japan \\ Correspondence should be addressed to Idnin Pasya; idninpasya@gmail.com
}

Received 28 February 2014; Revised 28 May 2014; Accepted 1 June 2014; Published 13 July 2014

Academic Editor: Mathini Sellathurai

Copyright (C) 2014 Idnin Pasya et al. This is an open access article distributed under the Creative Commons Attribution License, which permits unrestricted use, distribution, and reproduction in any medium, provided the original work is properly cited.

\begin{abstract}
This paper presents a joint direction-of-departure (DOD) and direction-of-arrival (DOA) estimation in a multiple-input multipleoutput (MIMO) radar utilizing ultra wideband (UWB) signals in detecting targets with fluctuating radar cross sections (RCS). The UWB MIMO radar utilized a combination of two-way MUSIC and majority decision based on angle histograms of estimated DODs and DOAs at each frequency of the UWB signal. The proposed angle estimation scheme was demonstrated to be effective in detecting targets with fluctuating RCS, compared to conventional spectra averaging method used in subband angle estimations. It was found that a wider bandwidth resulted in improved estimation performance. Numerical simulations along with experimental evaluations in a radio anechoic chamber are presented.
\end{abstract}

\section{Introduction}

The introduction of multiple-input multiple-output (MIMO) radar enables numerous improvements on the conventional single input single output radar system. The MIMO radar is typically defined as a radar system utilizing multiple transmitting and receiving antennas that are either widely distributed or colocated [1-4]. The former exploits independent path of transmit-receive pairs to increase the probability of detection [1, 2]. On the other hand, the latter uses orthogonality of transmitting signals to obtain an increased degree of freedom and parameter identifiability $[3,4]$, which are very useful in target localization applications.

Target localization in radar has been intensively studied in literatures since the early years of radar. In general, radar systems estimate the target position by means of trilateration or triangulation. Trilateration can be implemented by using a minimum of two stations; however, the localization resolution is limited by the signal bandwidth, and usage of multiple stations is required to avoid ambiguities (ghost targets). On the contrary, triangulation is based on the angles of targets observed from the radar stations, and hence it does not suffer from the bandwidth constraint. In MIMO radar, it is possible to jointly estimate the direction-of-departure (DOD) and direction-of-arrival (DOA) by implementing array processing at both of the transmitting and receiving arrays, as depicted in Figure 1. This makes it suitable for triangulation-based localization.

Numerous works on DOD and DOA estimation have been reported [5-8]. Nevertheless, these studies were mainly based on narrowband signal assumption. Target localization using the narrowband signal, however, was unstable due to fluctuation of target's radar cross section (RCS). In order to counter this problem, MIMO radars using multiple subcarriers and orthogonal frequency division multiplexing (OFDM) waveforms were proposed $[9,10]$. Those schemes, however, only discussed one-dimensional angle estimation of a single target and implementation of limited number of subbands. Utilization of wider signal bandwidth might be useful in localizing target with significantly small or severely 


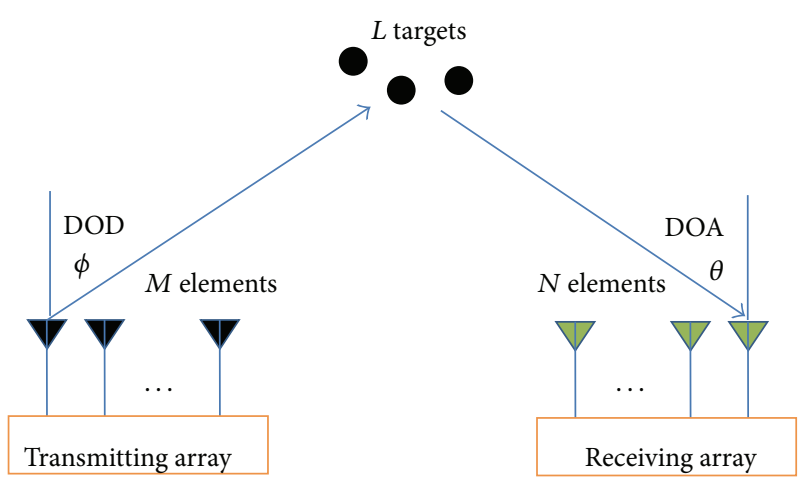

FIgURe 1: Overview of angle estimation in MIMO radar.

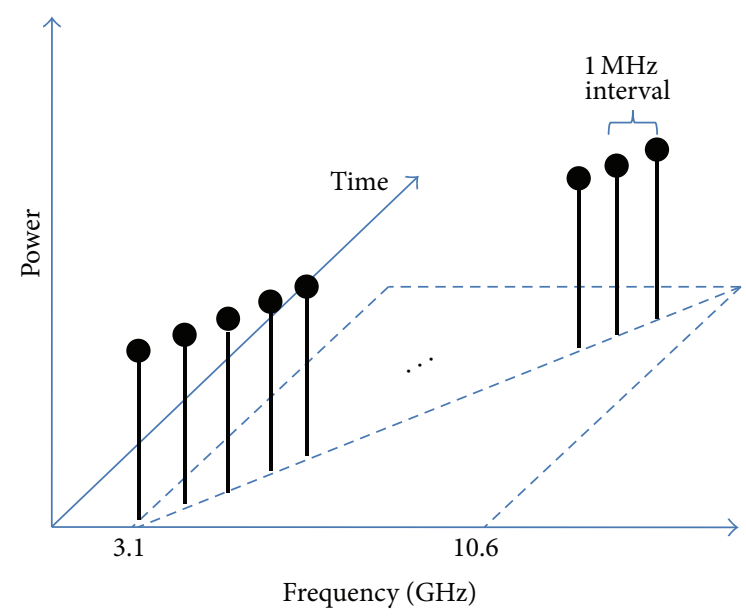

FIgURE 2: The proposed UWB signal for MIMO radar angle estimation.

fluctuating RCS. One of the most promising technologies with wideband capability is ultra wideband (UWB) systems. The usage of UWB signal for angle estimation in MIMO radar, however, has not been given much attention, limiting the study to medical imaging and through-the-wall radars [11, 12]. Application of conventional angle estimation methods (e.g., Capon, multiple signal classification (MUSIC), and estimation of signal parameters via rotational invariance (ESPRIT)) to a UWB signal is a challenge since those methods inherently assume narrowband signals.

The present authors proposed a joint DOD and DOA estimation in a UWB MIMO radar using the combination of a two-way MUSIC and angle histograms [13]. The basic idea in the proposed scheme was to treat the UWB signal as a summation of sinusoidal waves swept over the frequency band, and angle estimation was done at each of the frequency by means of two-way MUSIC [14]. The estimation results were then combined using majority decisions formulated using angle histograms [15]. However, the report only investigated estimation performance when detecting targets with a constant RCS, which was unlikely in actual environments.

In this paper, we evaluate the performance of the proposed algorithm in detecting targets with fluctuating RCS. Here, it will be shown that combining the estimation at different frequencies through majority decisions will overcome the problem of poor estimation when detecting fluctuating targets. A comparison between the proposed technique and the conventional spectrum averaging is also presented. Numerical simulations and experimental results are presented.

It is important to mention here that the RCS fluctuation problem has been continuously studied in the radar community. Until recently, special attention has been given to the subject in the case of MIMO radar, since the usage of MIMO configuration offers further degrees of freedom in the forms of spatial, frequency, and also waveform diversity. For example, the works in [16-18] employed spatial and waveform diversity in MIMO radars to increase the probability of detection and direction finding performance when detecting fluctuating target. The present study employs different approach from those reports since we focused on the utilization of frequency diversity. Studies regarding MIMO radars utilizing frequency diversity in detecting fluctuating RCS were studied, for instance, in $[19,20]$, where several subbands with substantially wide frequency spacing were used for angle estimation. This study differs from those works from a point of view that we employ the diversity among a large number of subbands throughout the frequency bandwidth of a UWB system to enhance target localization performance and specifically demonstrated the application to MIMO radar.

The remainder of this paper is organized as follows. The next section discusses the proposed algorithm. Section 3 describes the numerical simulations, and Section 4 explained the results of experimental evaluations. Finally, the concluding remarks are presented in Section 5.

\section{Proposed Scheme}

2.1. Proposed Joint DOD and DOA Estimation Scheme. Consider a MIMO radar with $M$ transmitting and $N$ receiving elements, illuminating $L$ uncorrelated targets located at the far field of transmit and receive arrays. At the transmit side, $M$ orthogonal UWB signals are emitted, and each consists of multiple sinusoidal waves swept over the UWB bandwidth. Here, we define the complex transmit signal waveform vector by $\mathbf{s}(t)=\left[\mathbf{s}_{1}(t), \ldots, \mathbf{s}_{M}(t)\right]$, where each term contains $K$ frequency components. The orthogonality between the transmit signals can be achieved through time division scheme, where each transmitting antenna emits the UWB signal in separate time slots. Figure 2 illustrates an example of the transmitting signal which uses 3.1 to $10.6 \mathrm{GHz}$ of sinusoidal waves in $1 \mathrm{MHz}$ intervals. The receiving signal of the $k$ th frequency component can be expressed by

$$
\mathbf{x}_{k}(t)=\left[\mathbf{a}_{t}(\phi) \otimes \mathbf{a}_{r}(\theta)\right] \mathbf{s}^{(k)}(t)+\mathbf{n}(t),
$$



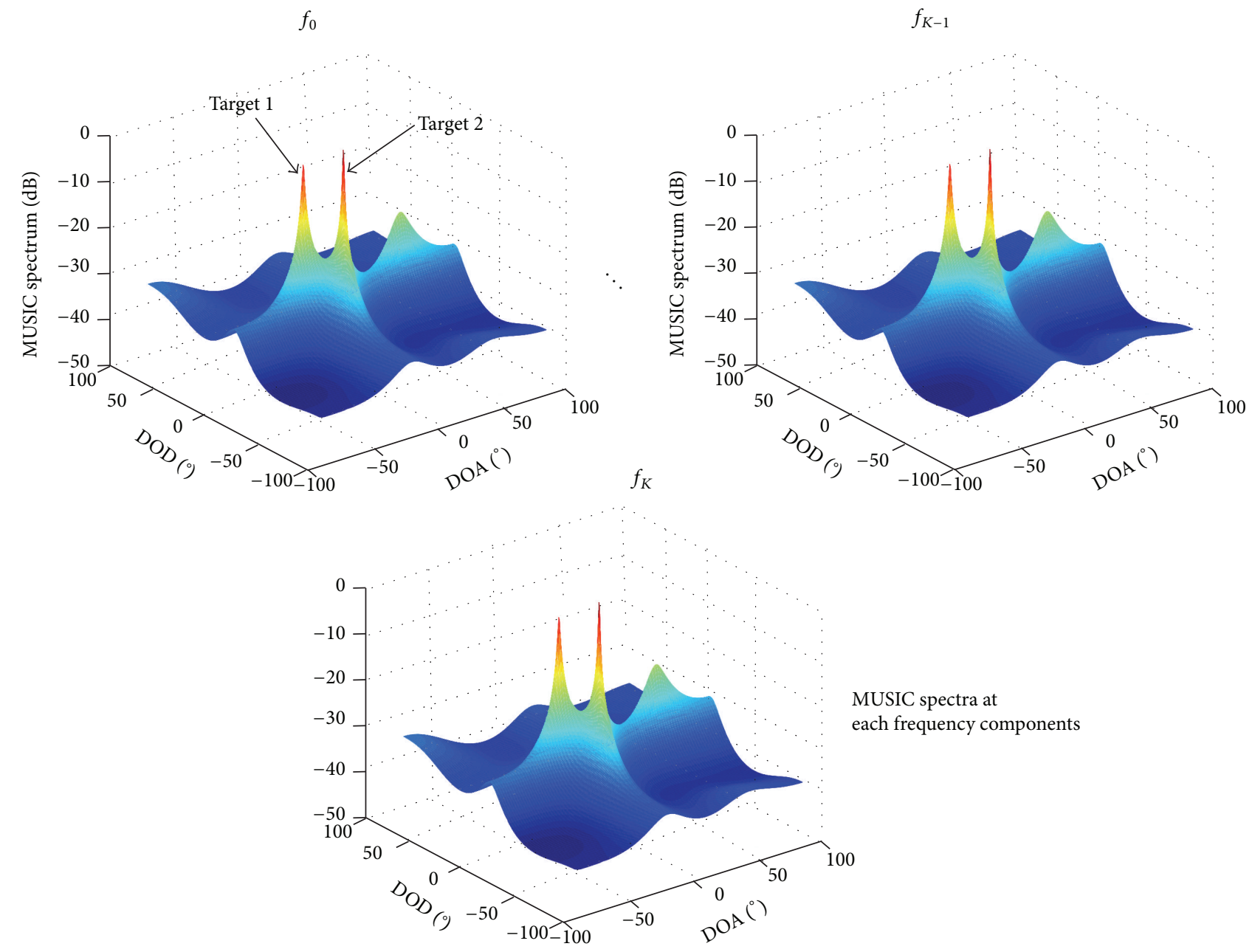

MUSIC spectra at

each frequency components
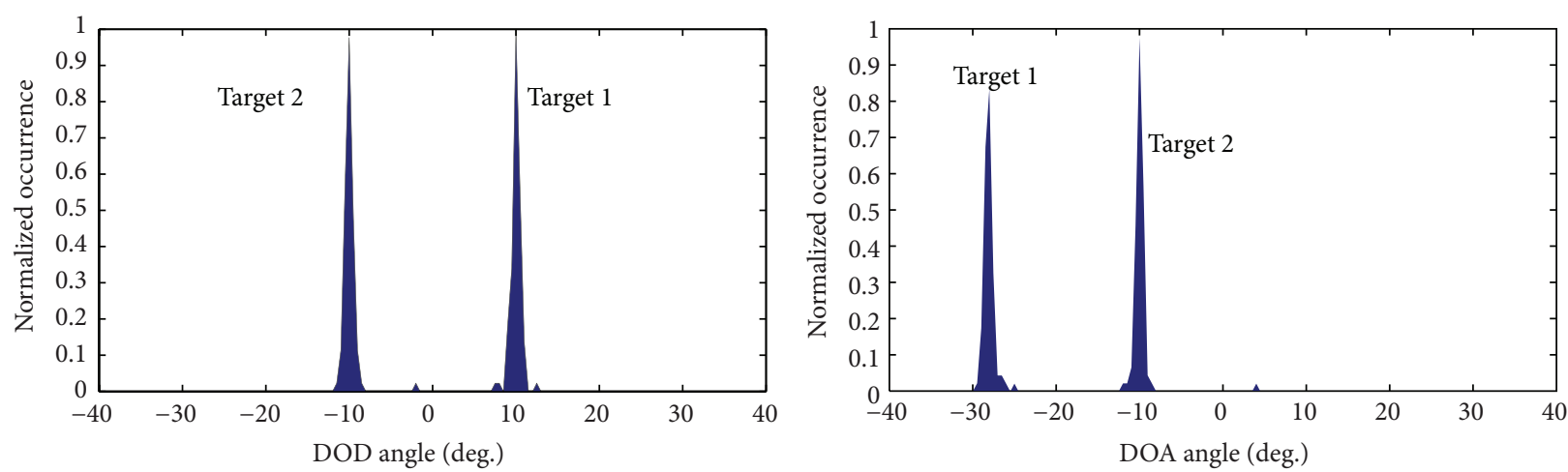

FIGURE 3: Formulation of angle histograms from two-way MUSIC spectra at each frequency component. 


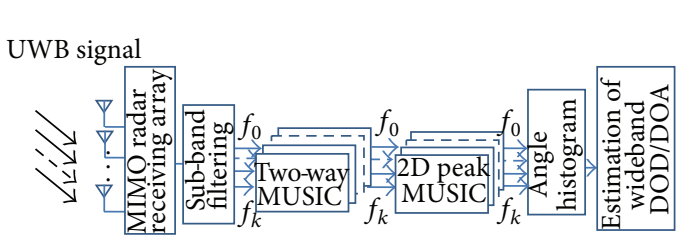

(a)

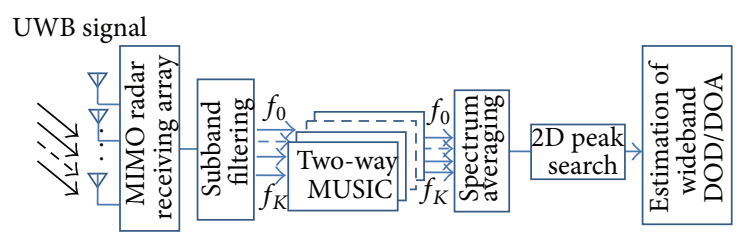

(b)

FIGURE 4: Block diagrams: (a) proposed and (b) conventional schemes.

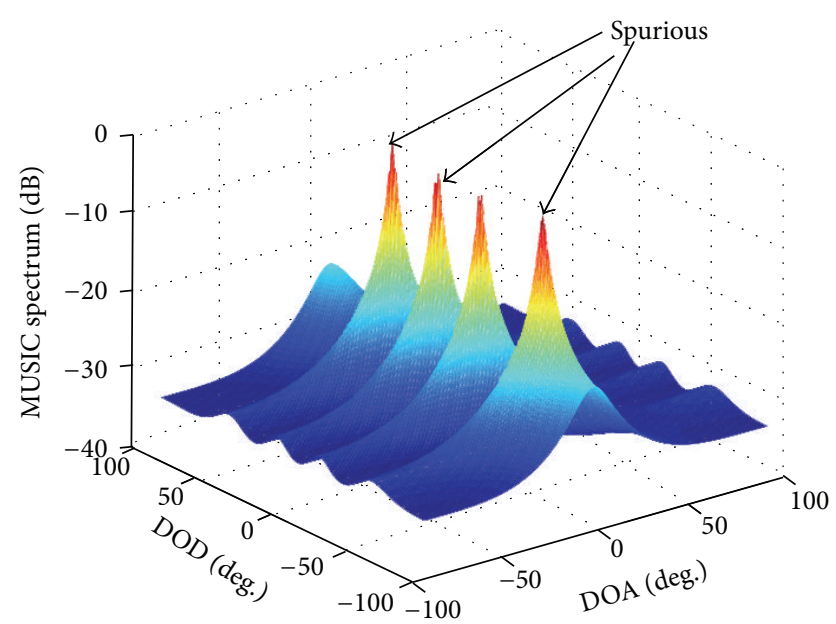

FIGURE 5: Example of spurious peaks in a simulated MUSIC spec$\operatorname{trum}\left(f_{k}=5.0 \mathrm{GHz}\right)$.

where $\otimes$ denotes the Kronecker product, $\mathbf{a}_{t}$ and $\mathbf{a}_{r}$ are the transmit and receive steering vectors, respectively, $\phi$ and $\theta$ are the corresponding transmit and receive angles, $\boldsymbol{s}^{(k)}(t)$ represents the $k$ th frequency component of the transmit signal vector $\mathbf{s}(t)$, and $\mathbf{n}(t)$ is the total additive white Gaussian noise. The receiving signal covariance matrix of the $k$ th frequency component is given by

$$
\mathbf{R}_{x x \_k}=E\left[\mathbf{x}_{k}(t) \cdot \mathbf{x}_{k}(t)^{H}\right],
$$

where $E[\cdot]$ is the ensemble average and $[\cdot]^{H}$ represents the conjugate transpose operation. Here, singular value decomposition (SVD) of the covariance matrix gives

$$
\mathbf{R}_{x x \_k}=\mathbf{E}^{(k)} \mathbf{V}^{(k)} \mathbf{E}^{(k)^{H}}
$$

where $\mathbf{V}^{(k)}$ is a diagonal matrix whose diagonal elements contain the signal and noise eigenvalues for the $k$ th frequency and $\mathbf{E}^{(k)}$ is the corresponding eigenvectors of the signal and noise components. The two-dimensional spatial MUSIC spectrum at the $k$ th frequency component can be constructed using the function

$$
P_{\mathrm{MU} \_k}(\phi, \theta)=\frac{1}{\left[\mathbf{a}_{t}(\phi) \otimes \mathbf{a}_{r}(\theta)\right]^{H} \mathbf{e}_{N}^{(k)} \mathbf{e}_{N}^{(k)}\left[\mathbf{a}_{t}(\phi) \mathbf{a}_{r}(\theta)\right]},
$$

where $\mathbf{e}_{N} \mathbf{e}_{N}^{H}$ is the noise eigenvectors obtained from the eigendecomposition of the receive signal covariance matrix in (2). Here, we have the $L$ largest peaks which correspond to the DOD and DOA of the targets at each frequency component. The wideband DOD and wideband DOA are decided by taking the majority of the estimated angles among all the estimates at each frequency. This is denoted by "majority decision" in this study. The majority decision is formulated by initially combining DOD and DOA estimates at the $K$ frequencies into a vector and arranging them in the form of angle histograms [13], as depicted in Figure 3. The angle histogram can be viewed as a function of angle $i$ from $-90^{\circ}$ to $90^{\circ}$ at intervals of, for example, $0.5^{\circ}$. The normalized number of occurrence of the peak angle is then given by

$$
\widehat{r}^{(i)}=\frac{1}{z} p^{(i)}
$$

where $p^{(i)}$ is the number of occurrences of the angle $i$ and $z$ is the normalized coefficient given by

$$
z=\arg \max \widehat{r}^{(i)} .
$$

The majority decision is obtained by searching the peak of the histogram. As a benchmark, the performance of the proposed scheme will be compared with the conventional spectrum averaging method [21] used in existing subband processing scheme for angle estimation. The spectrum averaging method adopts an approach which takes the average of the estimated MUSIC spectra of all $K$ frequency components:

$$
P_{\mathrm{MU} \_a v e}(\phi, \theta)=\frac{1}{K} \sum_{k=1}^{K} P_{\mathrm{MU} \_k}(\phi, \theta) .
$$

Then the wideband DOD and DOA are estimated from the $L$ largest peaks of $P_{\mathrm{MU} \text { ave }}$. We will demonstrate in Section 3 that the majority decision technique performs better than the spectrum averaging method. Figure 4 depicts the block diagrams of the proposed and conventional schemes.

2.2. MIMO Radar Array Configuration Used in this Study. The main advantage of a MIMO radar system is that the degrees of freedom can be enhanced by using the concept of virtual array $[22,23]$. When orthogonal signals were transmitted from different antennas, the back scatter returns of each orthogonal signal will carry independent phase information that forms a new virtual array steering vectors at the receiver. 

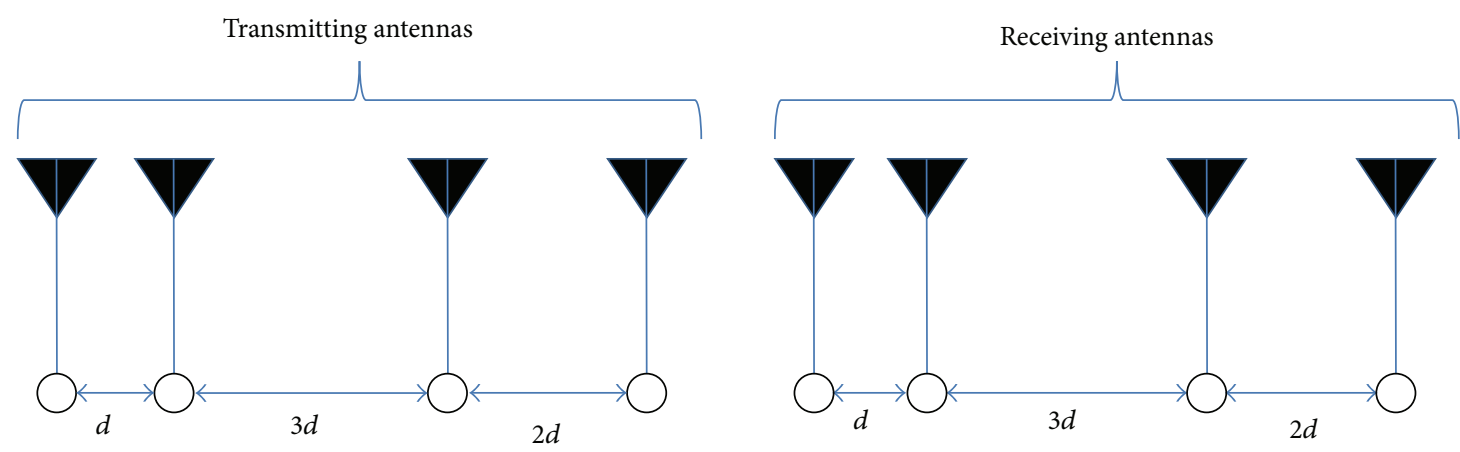

Figure 6: Nonuniform array configuration used in the study $(d=15 \mathrm{~mm})$.

The virtual array can be characterized by convolution of the transmitting and receiving antenna positions [22]. Given a MIMO radar using $M$ transmitting and $N$ receiving antennas, it is possible to form a full virtual array with nonoverlapping $M N$ elements, by optimizing the antenna positions. Larger numbers of $M$ and $N$ contribute to construction of longer virtual array, which means further enhancement in angle estimation performance. Thus, from a signal processing point of view, it is generally important to use a larger virtual array. However, in practical applications, it is often beneficial to limit the number of antennas for the sake of cost and space. In this study, we demonstrate the proposed algorithm using a $4 \times 4$ MIMO array and discuss the performance within that limitation.

A full $M N$ virtual array can be constructed, for example, by using transmitting antennas with spacing of $N d$ and receiving antennas with $d$ spacing [23], where $d$ is the distance equal to the half wavelength.

However, as shown in Figure 5, utilizing a two-way MUSIC using this array configuration resulted in spurious peaks in the MUSIC spectrum, since the receiving array's spacing is much larger than the distance of half wavelength. This problem can be overcome by limiting the scan range, but in the cost of narrower coverage area.

In this study, we employed a nonuniform array configuration as shown in Figure 6 to reduce the spurious peaks. This array configuration was chosen due to the minimum number of redundant elements that could be obtained in the virtual MIMO array. Refer to the Appendix for further explanation. The basic antenna spacing of $d=15 \mathrm{~mm}$ (corresponds to half wavelength of $10 \mathrm{GHz}$ ) was used. Figure 7 depicts the resulting MUSIC spectrum using the nonuniform array, where we could observe that the spurious peaks were eliminated, and the resulting spectrum peak sharpness is comparable to a full $M N$ array with limited range scan. The authors have previously reported in [13] that this array configuration outperforms the conventional uniform linear array.

2.3. Complexity Analysis. This subsection presents the analysis of the computational complexity of the proposed scheme. The computational burden of a conventional 2D-MUSIC has

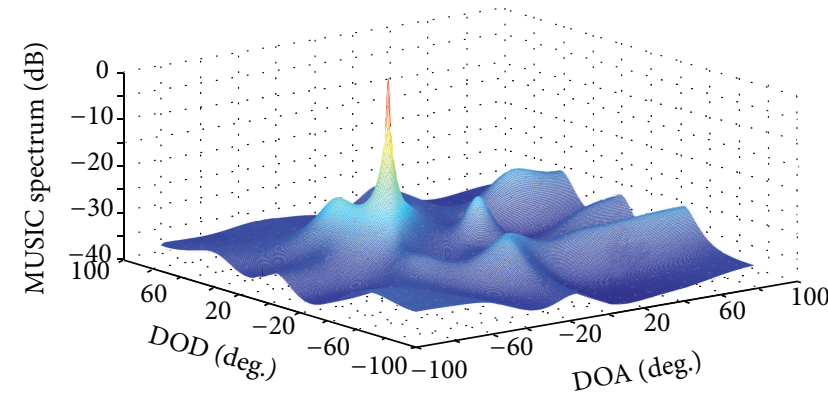

FIGURE 7: Example of a MUSIC spectrum using the nonuniform array configuration $\left(f_{k}=5.0 \mathrm{GHz}\right)$.

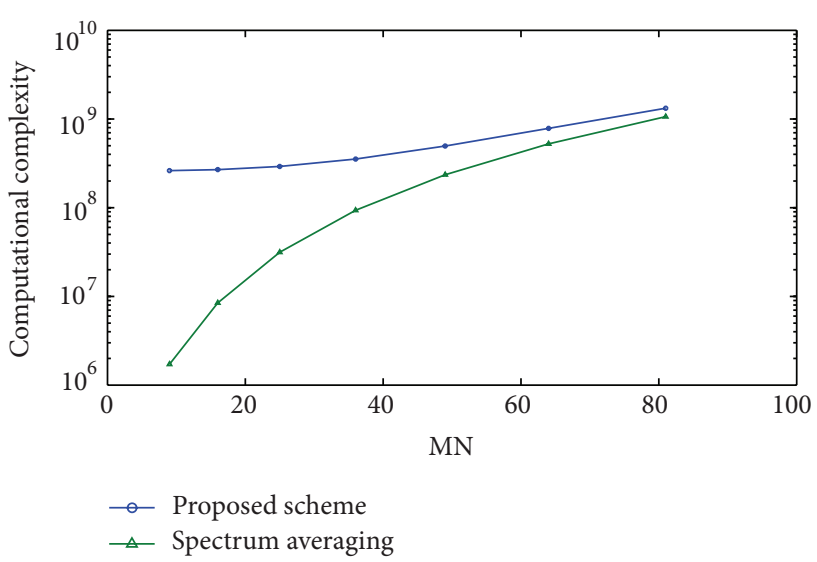

FIGURE 8: Computational complexity of the proposed scheme against $M N$ antennas $(K=1000, L=2)$.

been reported in literatures, such as in [5]. For the sake of clarity, we broke down the complexity analysis of the proposed scheme in terms of SVD operation and searching algorithm computational costs, expressed using the $O$ notation [24]. 
TABLE 1: Simulation parameters.

\begin{tabular}{lc}
\hline Parameters & Description \\
\hline Number of transmitting antennas, $M$ & 4 \\
Number of receiving antennas, $N$ & 4 \\
Number of targets, $L$ & 2 \\
Signal to noise ratio, SNR & $15 \mathrm{~dB}$ \\
Number of snapshots & 50 \\
Target positions & $\left(\phi_{1}, \theta_{1}\right)=\left(10^{\circ},-28^{\circ}\right)$ \\
& $\left(\phi_{2}, \theta_{2}\right)=\left(-10^{\circ},-10^{\circ}\right)$ \\
Type of targets & Fixed point targets or \\
& Weibull targets \\
\hline
\end{tabular}

The conventional spectrum averaging method [21] was also analyzed for comparison.

Considering the dimensions of the covariance matrix $u \times v$ and the total $K$ frequency components used, applying SVD operation on the receiving covariance matrix generally costs $O\left(K\left\{u^{2} v+v^{3}\right\}\right)$. Here, from the receiving covariance matrix formulation, $u$ and $v$ are given by $M^{2}$ and $N^{2}$, respectively. This is the same for both the proposed and the spectrum averaging schemes. In terms of peak search operation, the proposed scheme performs two-dimensional peak search on the MUSIC spectrum, which costs $O\left(i^{2} K L\right)$, where $i$ is the number of angle bins during search operations. In addition, the majority decision routine costs $O(2\{i+K\})+O(2\{i L\})$, where the first term corresponds to the histogram formulation of $K$ total estimates, and the second term represents the one-dimensional peak search to identify the angles of $L$ targets. On the other hand, the computational burden of the spectrum averaging method is $O(K)+O\left(i^{2} L\right)$, corresponding to the spectrum averaging and two-dimensional peak search of the averaged spectrum, respectively.

The computational complexity against $M N$ antennas is plotted in Figure 8. It was shown that the proposed scheme marked larger computational burden than the spectral averaging method, particularly when the number of antennas is small. However, the difference of complexity is reduced with increasing number of antennas and converges when $M N$ approaches 81 . This is because the cost of SVD operation becomes dominant with larger dimension of receiving covariance matrix, thus resulting in similar order of total computational complexity in both methods.

\section{Simulation Results}

The proposed algorithm was simulated according to the parameters listed in Table 1. As mentioned in the previous sections, the UWB signal considered in the proposed scheme contains multiple sinusoidal ranging from 3.1 to $10.6 \mathrm{GHz}$ at $1 \mathrm{MHz}$ intervals. Here, selection of the start and stop frequencies within the frequency range determines the total

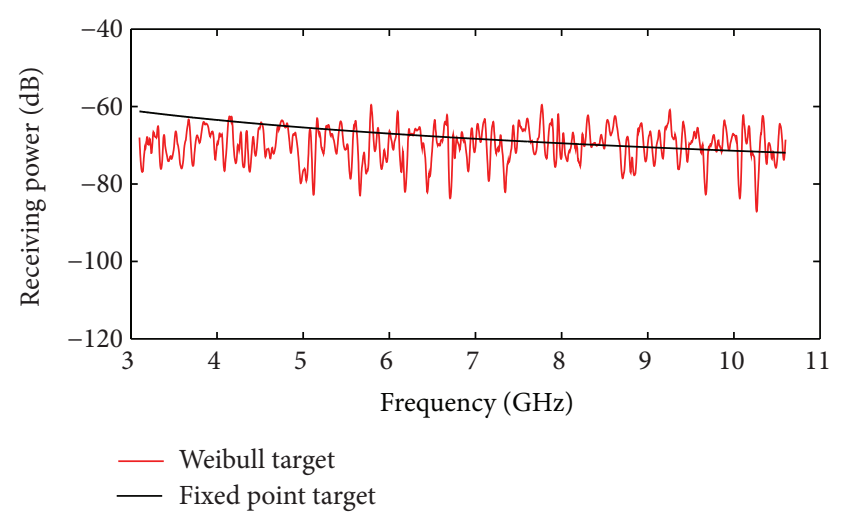

FIgURE 9: Simulated RCS of a simulated Weibull target in comparison with a fixed point target considering propagation loss in frequency domain.

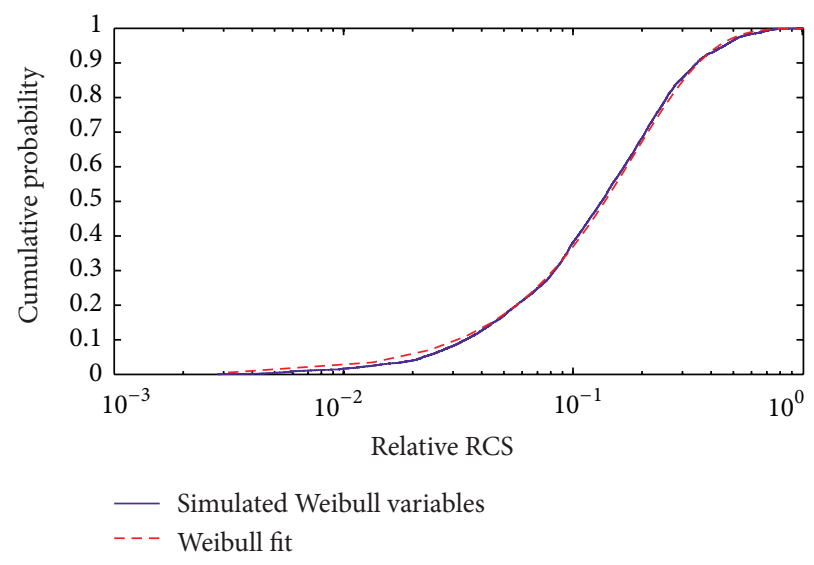

FIgURE 10: Cumulative distribution of the simulated RCS of a Weibull target in frequency domain.

bandwidth of the signal used. The signal bandwidth is defined by

$$
\text { Bandwidth }=f_{H}-f_{L} \text {, }
$$

where $f_{H}$ and $f_{L}$ are the highest and lowest frequency components, respectively. The simulations will be conducted while varying the signal bandwidth from 10 to $1000 \mathrm{MHz}$, at different center frequencies, $f_{c}$, for example, from $3.6,5$, and 8.4 to $10 \mathrm{GHz}$.

Two different scenarios were simulated, where the MIMO radar was detecting either a fixed point target or a target with fluctuating RCS. The fixed point target was modeled with a constant RCS, normalized by the free space propagation loss coefficient in each frequency. The targets with fluctuating RCS were modeled by Weibull distribution, since it was shown in literatures that measured RCS of complex targets such as automobiles and small cars follows Weibull distribution [25]. The measurement data also showed that at a given observation angle, the RCS against frequency (7 $\mathrm{GHz}$ bandwidth) also follows similar distribution. The targets considered in the simulation were modeled to have 

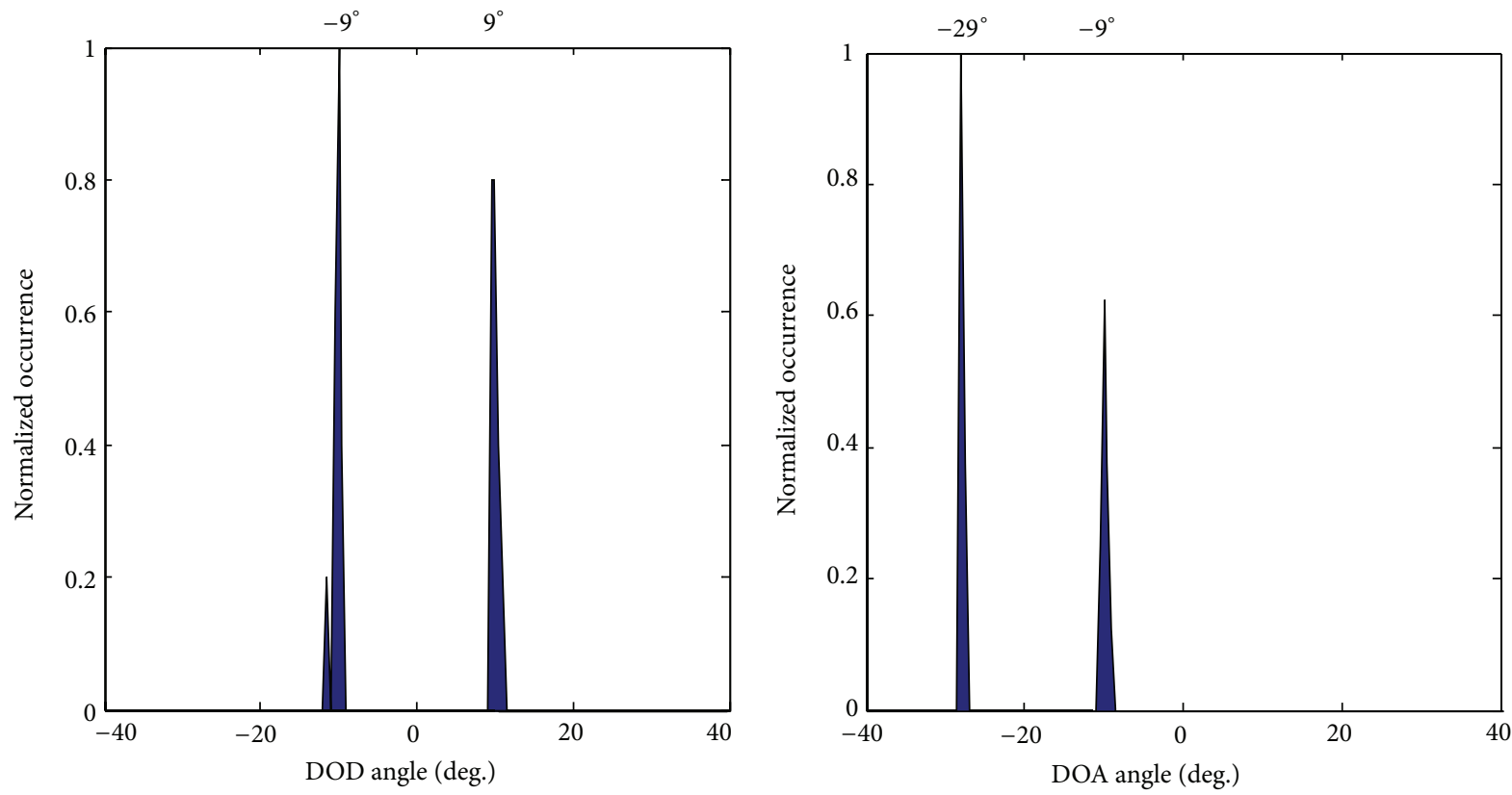

(a)
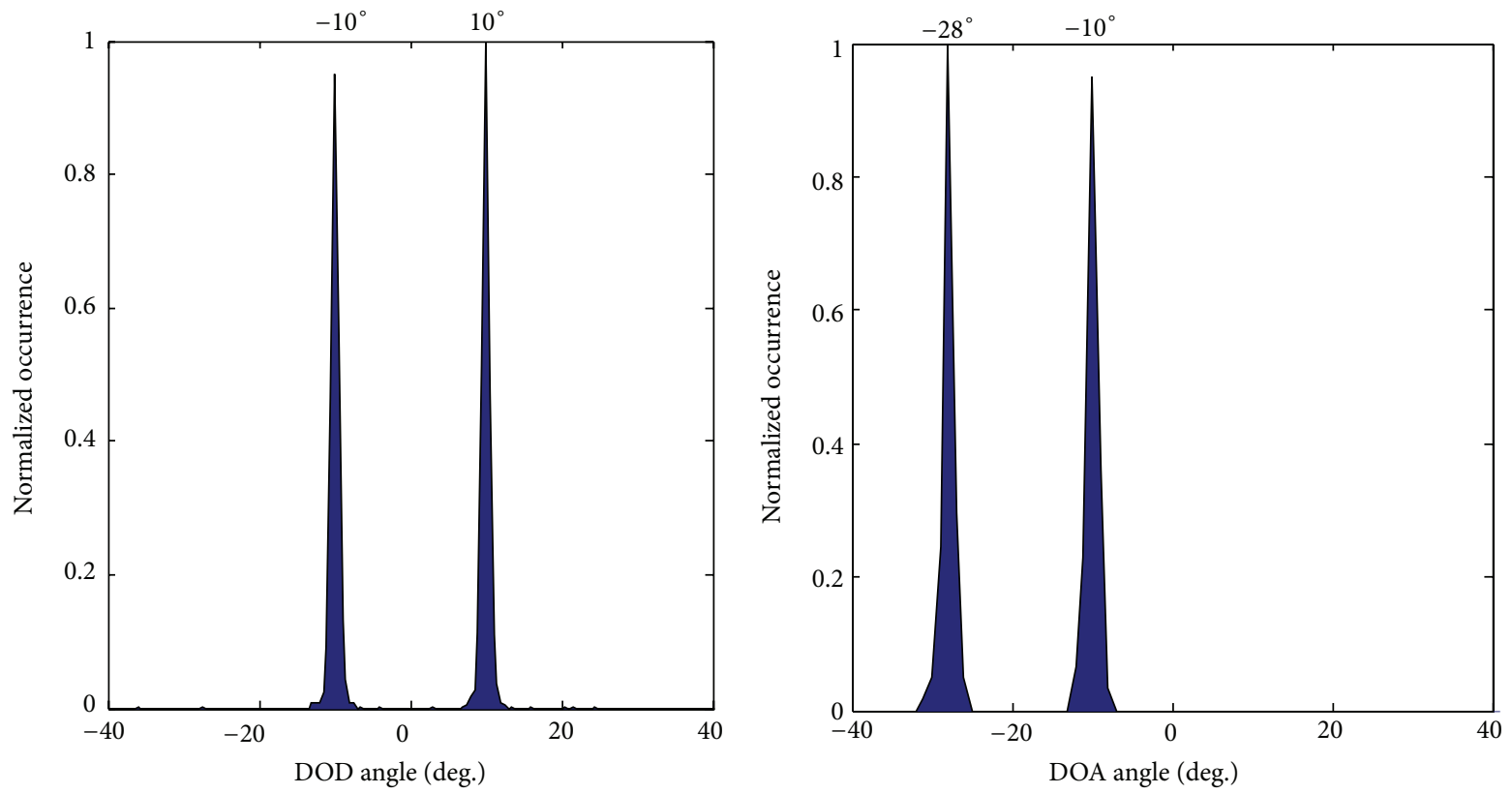

(b)

FIGURE 11: Angle histograms of estimated DODs and DOAs in detecting fixed point targets using a signal bandwidth of (a) 50 and (b) $1000 \mathrm{MHz}$.

Weibull distributed RCS throughout the UWB frequency range, whose cumulative distribution function is given by

$$
f(x)= \begin{cases}1-\exp ^{-(x / a)^{b}}, & x \geq 0 \\ 0, & x<0\end{cases}
$$

where $a$ and $b$ are the shape and scale parameters, respectively. The values of $a$ and $b$ were selected so as to equal the RCS medians of the Weibull and fixed point targets. Figure 9 plots the RCS versus frequency (expressed in signal power), and Figure 10 depicts the corresponding cumulative distribution. We demonstrate the performance of the proposed scheme when detecting 2 targets located at $\left(\phi_{1}, \theta_{1}\right)=\left(10^{\circ}\right.$, $\left.-28^{\circ}\right)$ and $\left(\phi_{2}, \theta_{2}\right)=\left(-10^{\circ},-10^{\circ}\right)$. The signal to noise ratio (SNR) was $15 \mathrm{~dB}$, and the number of snapshots was 50 .

Figure 11 shows the examples of angle histograms at $f_{c}=$ $3.6 \mathrm{GHz}$, when the MIMO radar was detecting the fixed point targets. It can be observed that the angle histograms 

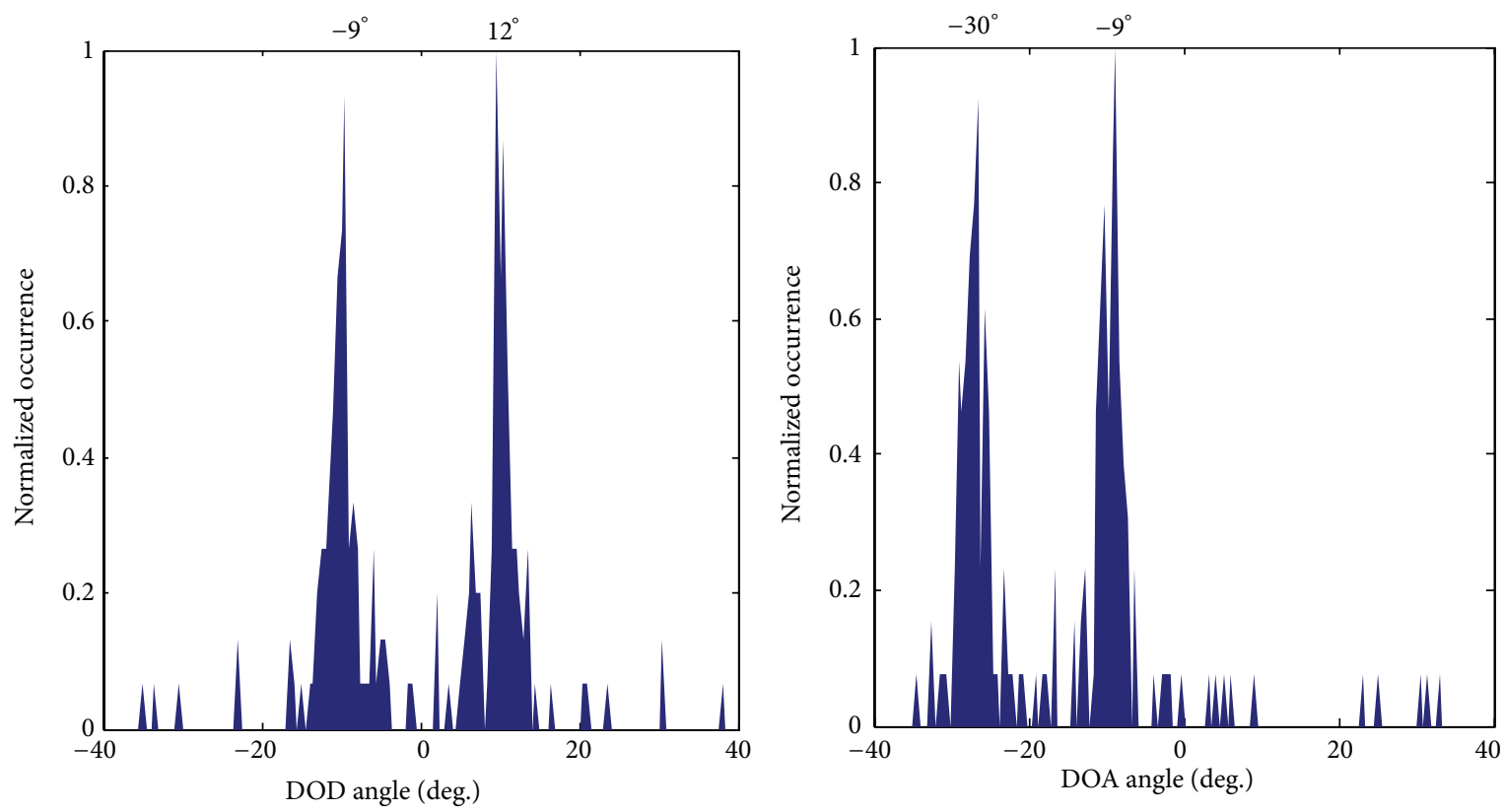

(a)
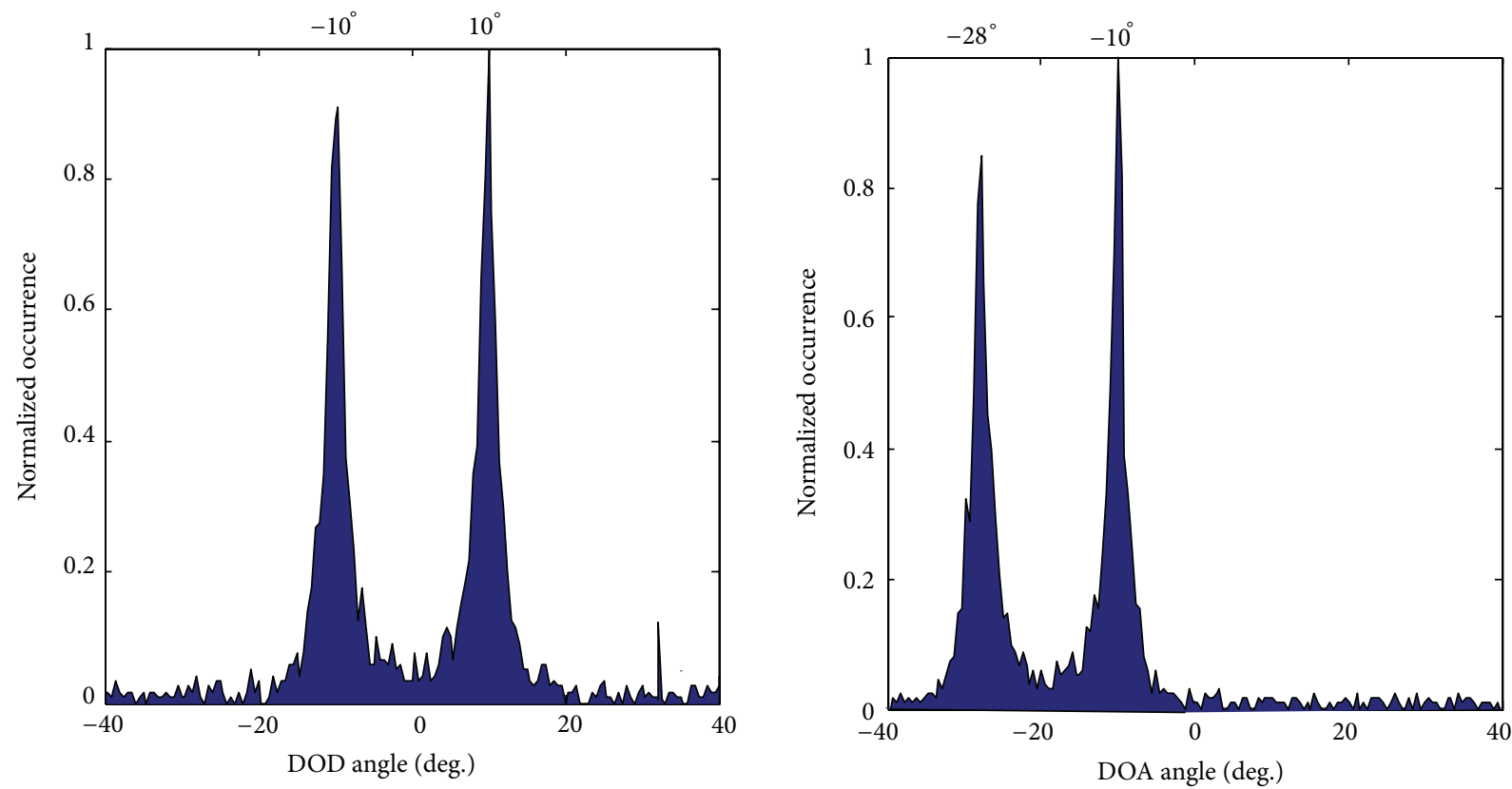

(b)

FIgURE 12: Angle histograms of estimated DODs and DOAs in detecting Weibull targets using a signal bandwidth of (a) 50 and (b) 1000 MHz.

were more or less similar when using either $1000 \mathrm{MHz}$ or $50 \mathrm{MHz}$ of signal bandwidths. Both cases marked sharp histogram peaks, which corresponds to the respective targets. This deduced that, when detecting fixed point targets, the bandwidth has minimal effects on the angle estimation performance. The estimation using a $50 \mathrm{MHz}$ signal bandwidth, however, yielded an error of $1^{\circ}$. On the other hand, the angle estimation of the Weibull targets marked outstanding outliers in the angle histograms, particularly when using a $50 \mathrm{MHz}$ signal bandwidth, as depicted in Figure 12(a). These outliers were attributable to spurious peaks in the MUSIC spectrum due to small RCS values at certain frequencies. However, taking the majority decisions from the histograms, the targets were successfully estimated within $2^{\circ}$ of estimation error. It was also shown in Figure 12(b) that, by taking a larger bandwidth, for example, $1 \mathrm{GHz}$, the peaks of the outliers were suppressed compared to using a $50 \mathrm{MHz}$ signal bandwidth. 

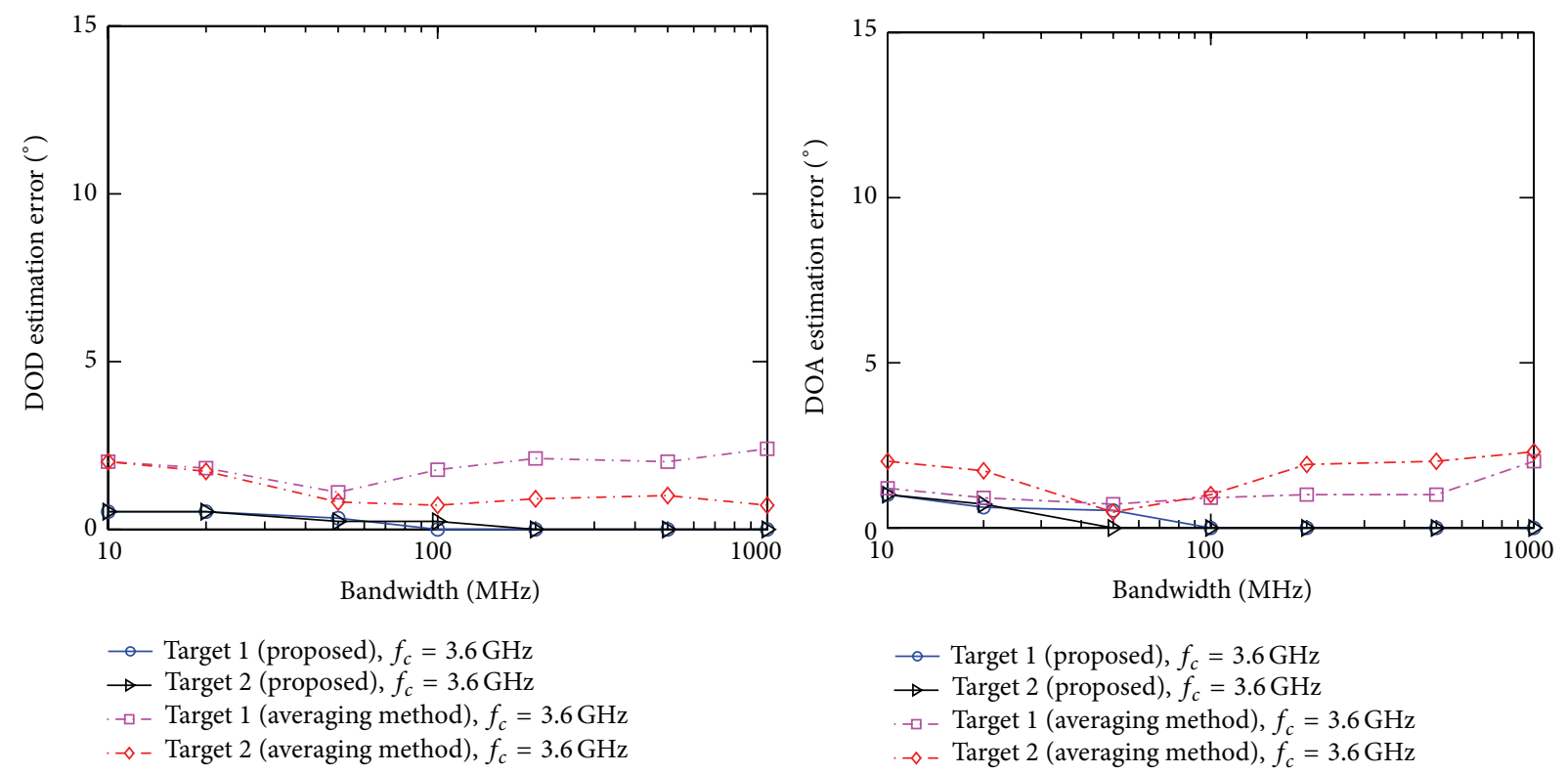

(a)
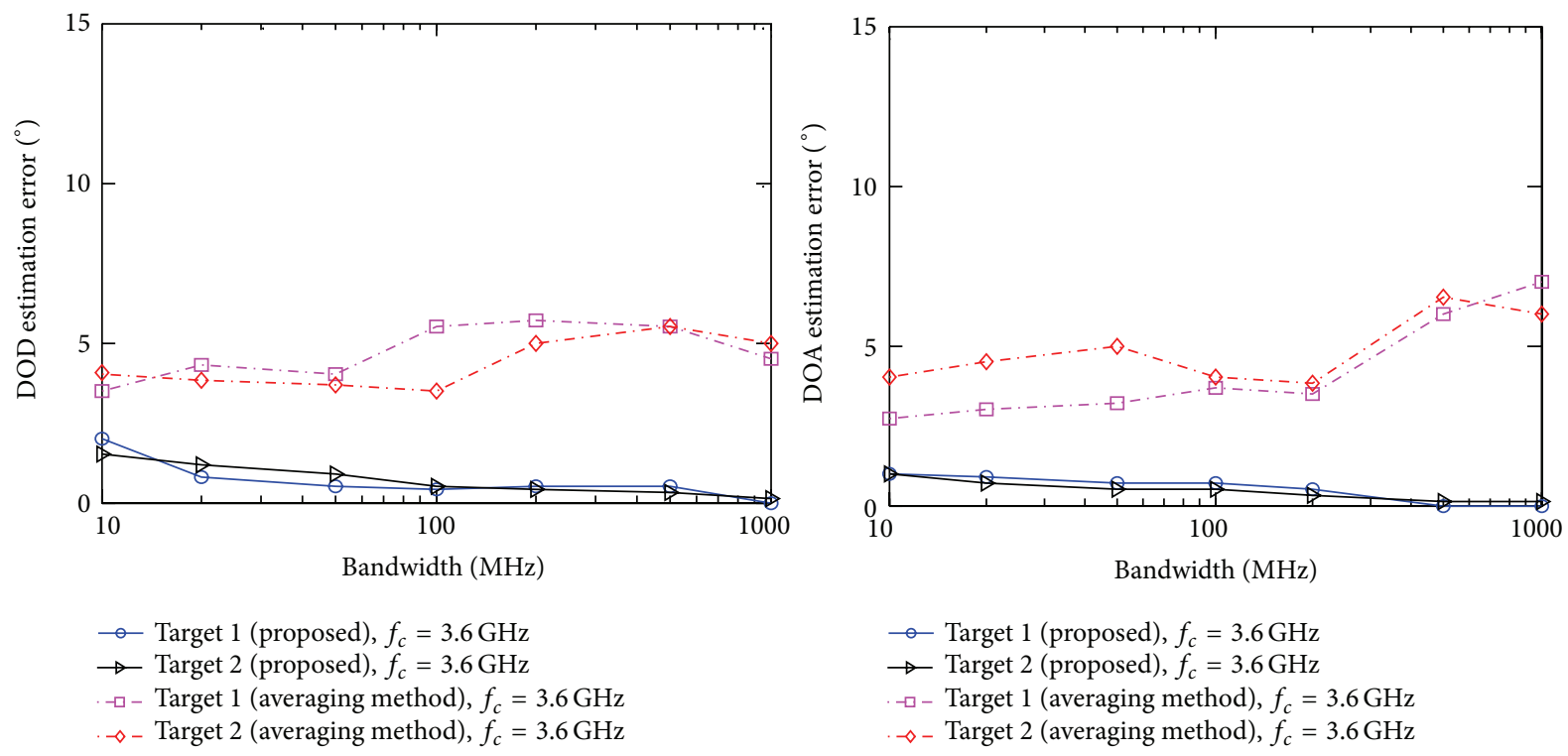

(b)

FIGURE 13: Estimation errors against bandwidth: (a) fixed point and (b) Weibull targets.

The performance of the proposed scheme was evaluated in terms of estimation error, defined by

$$
\begin{aligned}
& \text { Error }_{\mathrm{DOD}}=\frac{1}{W} \sum_{w=1}^{W}\left|\phi-\phi_{\mathrm{est}}\right|, \\
& \text { Error }_{\mathrm{DOA}}=\frac{1}{W} \sum_{w=1}^{W}\left|\theta-\theta_{\text {est }}\right|,
\end{aligned}
$$

where $W$ is the number of independent trials and $\phi_{\text {est }}$ and $\theta_{\text {est }}$ are the estimated DOD and DOA, respectively. The estimation error against signal bandwidth using $f_{c}=$ 3.6 GHz was plotted in Figure 13, comparing the performance when detecting the fixed point and Weibull targets with 50 independent trials and SNR $=15 \mathrm{~dB}$. Comparison with a conventional spectra averaging method [21] was also presented. It can be observed that the proposed scheme yielded improved estimation performance when taking larger signal bandwidth. This was true when detecting both types 

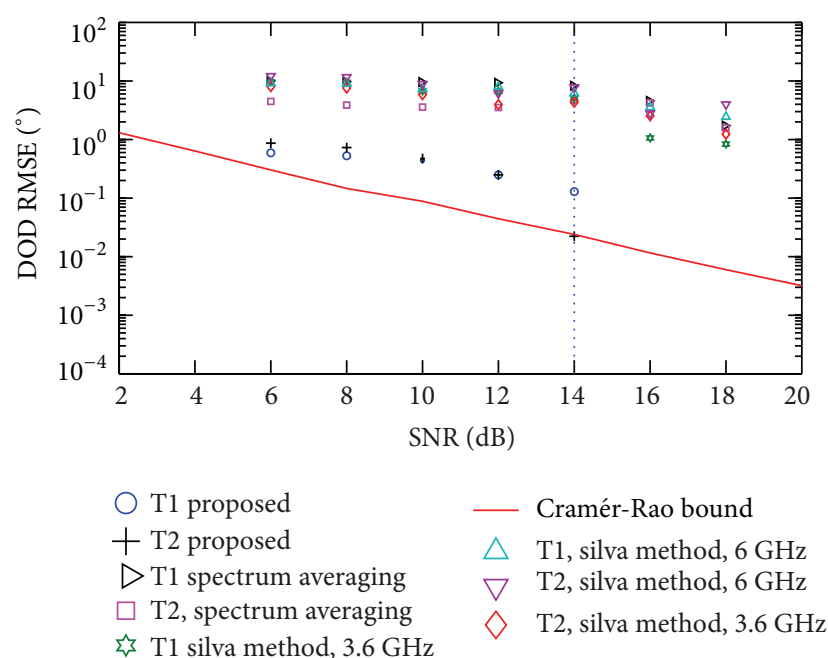

(a)

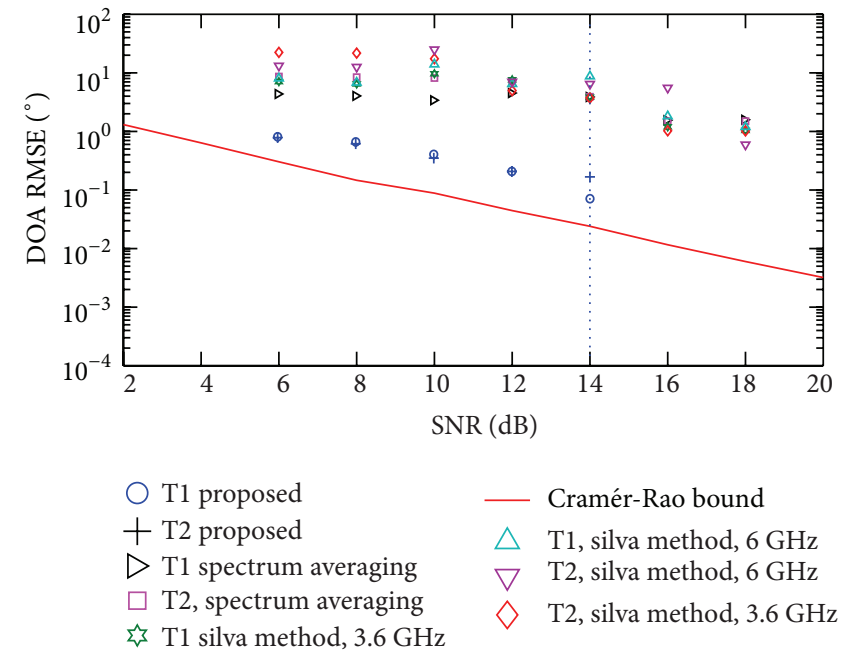

(b)

FIGURE 14: RMSE performance of the proposed scheme: (a) DOD and (b) DOA.

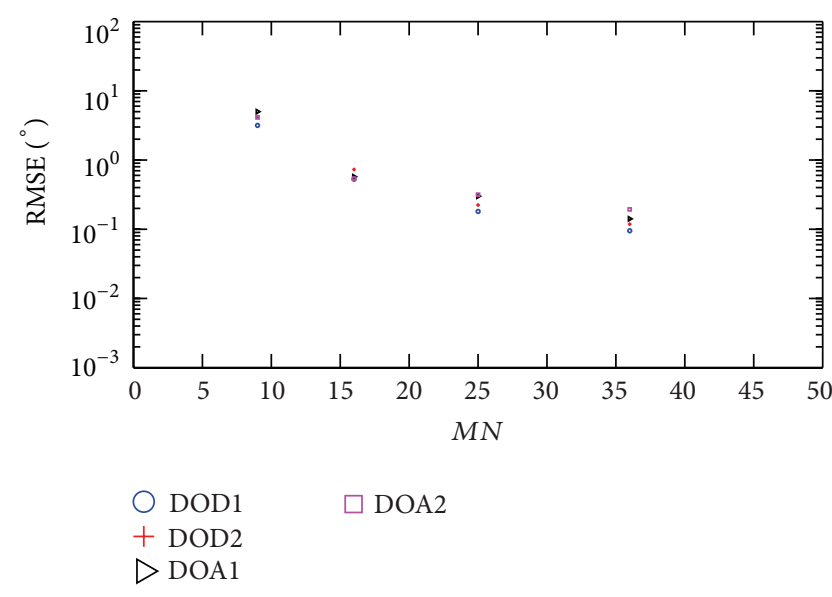

FIGURE 15: RMSE of the proposed scheme against $M N$ (bandwidth $=1000 \mathrm{MHz}, L=2, \mathrm{SNR}=8 \mathrm{~dB}$, and target $=$ Weibull).

of targets; however, the improvement was more significant in the case of Weibull targets. On the other hand, the estimation error increased with larger bandwidth when conventional spectra averaging method was applied. This is attributed to large number of outliers which significantly influenced the averaging result. This result demonstrated the effectiveness of the proposed method in detecting targets with severely fluctuating RCS.

The performance of the proposed scheme in terms of root mean square error (RMSE) against SNR is plotted in Figure 14. Performance of the spectrum averaging method and another conventional method by de Silva and Seow [14] was also presented for benchmarking purposes. Simulation was carried out assuming that all methods use a $4 \times 4$ MIMO nonuniform array detecting two Weibull targets. The performance of the proposed and spectrum averaging schemes was shown when they are using a $1000 \mathrm{MHz}$ bandwidth with $f_{c}=3.6 \mathrm{GHz}$, while Silva's method was presented at several frequencies since it is a narrowband-based angle estimation. The performance bound calculated from the Cramér-Rao bound (CRB) when the DOD and DOA of a stationary target are estimated individually $[26,27]$ was also plotted for reference.

We could observe that the proposed scheme marked the best performance, which was the nearest to the CRB, and produced no estimation error when the SNR exceeds $14 \mathrm{~dB}$. It was also shown that the performance of Silva method depended on the frequency for the targets with fluctuating RCS.

The impact of a number of antennas on the performance of the proposed scheme was plotted in Figure 15. The RMSE was computed in 50 independent trials against $M N$, when using a signal bandwidth of $1000 \mathrm{MHz}$ and $\mathrm{SNR}=8 \mathrm{~dB}$. Specific cases of $M=N=3,4,5$, and 6 were simulated. It is shown in the figure that increasing number of antennas yielded improvement in RMSE performance, even in the low SNR condition.

\section{Experimental Results}

Experiments were conducted to verify the results of the numerical simulations. The measurements were done in a radio anechoic chamber, using a measurement setup illustrated in Figure 16. The setup consists of a vector network analyzer (VNA) and GPIB-controlled scanners which were used to virtually construct the MIMO arrays at both transmitting and receiving sides. The VNA was used to generate UWB signals from 3.1 to $10.6 \mathrm{GHz}$ in $1 \mathrm{MHz}$ intervals, which were 


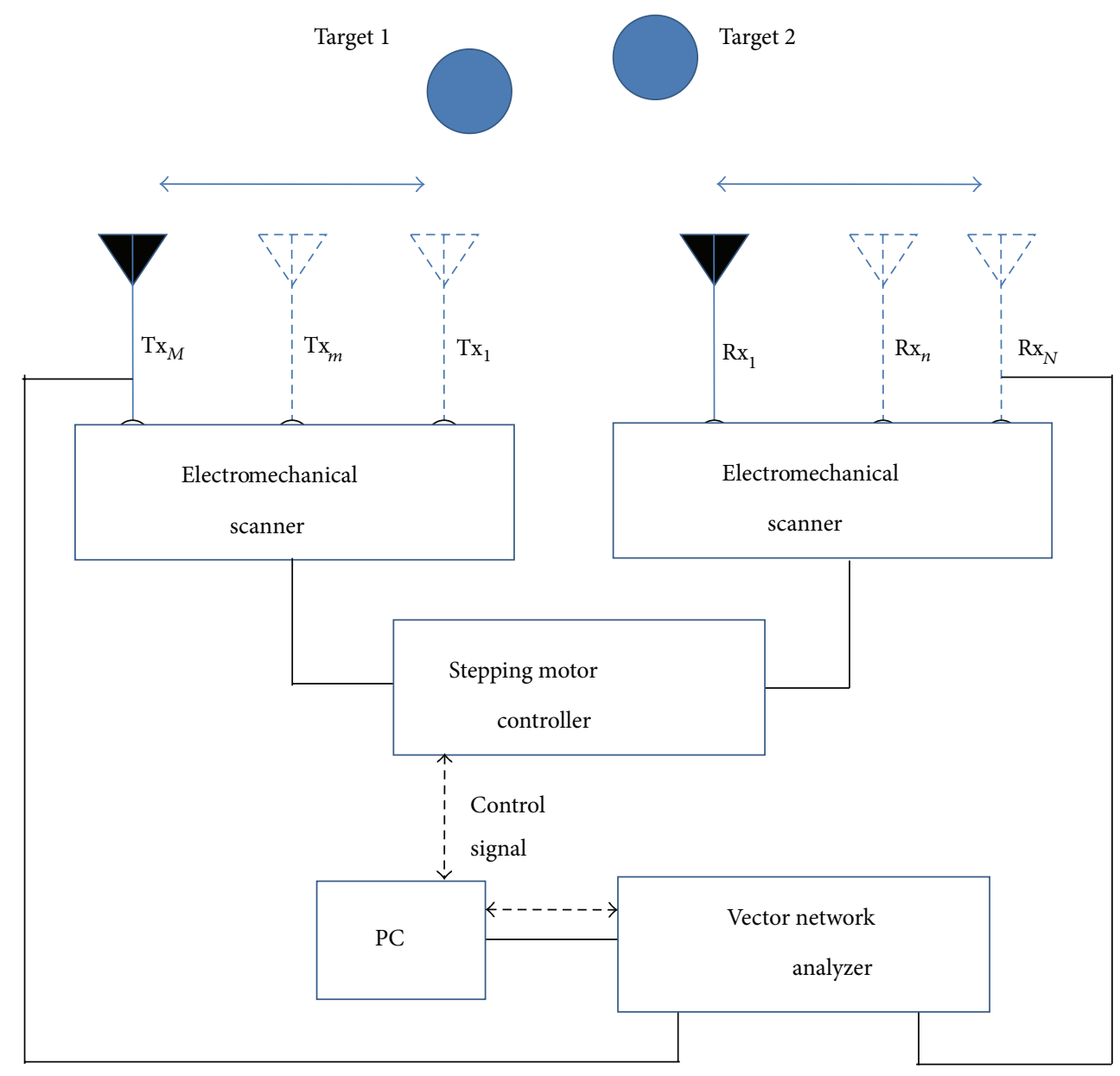

FIGURE 16: Measurement setup.

used as the transmitting signal. The VNA was adopted due to its good calibration functions and time gating capability to cancel out direct coupling effects between transmitting and receiving antennas. Wideband horn antennas with average gain of $12.5 \mathrm{dBi}$ were used as both transmitting and receiving antennas. The SNR of the system is defined as the ratio of the receiving signal average power to the thermal noise.

The setup was used to localize two targets positioned at $\left(\phi_{1}, \theta_{1}\right)=\left(10^{\circ},-28^{\circ}\right)$ and $\left(\phi_{2}, \theta_{2}\right)=\left(-10^{\circ},-10^{\circ}\right)$, similar to the condition in previous simulations. Two types of targets were used, which were conductive spheres and complex shaped targets, both fabricated using polystyrenes and aluminum foil. The conductive spheres have $20 \mathrm{~cm}$ of diameters which yield RCS of approximately $-10 \mathrm{dBm}^{2}$. The complex targets were constructed so that they yield a fluctuating RCS in the frequency domain. An example of the complex target is shown in Figure 17. Figure 18 shows the measured frequencydomain data of the complex targets, where $\psi$ is the monostatic angle of observation. From the figure, we could observe that the receive signal fluctuates severely against frequency, compared to that of sphere targets. The complex targets

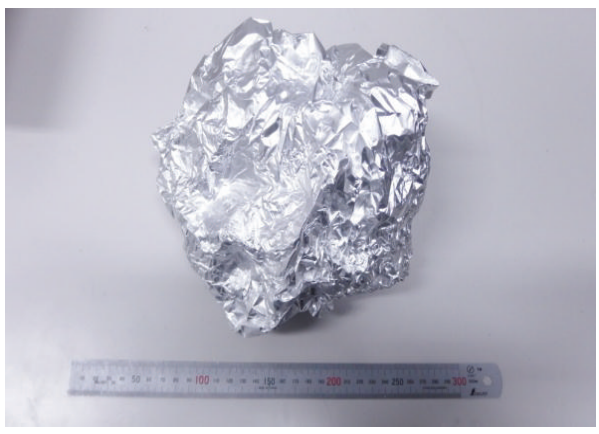

FIGURE 17: Example of a fabricated complex target.

marked several frequency regions with fluctuations of more than $15 \mathrm{~dB}$ at different angles, compared to sphere targets with only $5 \mathrm{~dB}$ of maximum fluctuations. The targets were positioned at $2.5 \mathrm{~m}$ from the baseline of the transmitter and receiver. The measurement scenario is depicted in Figure 19. 


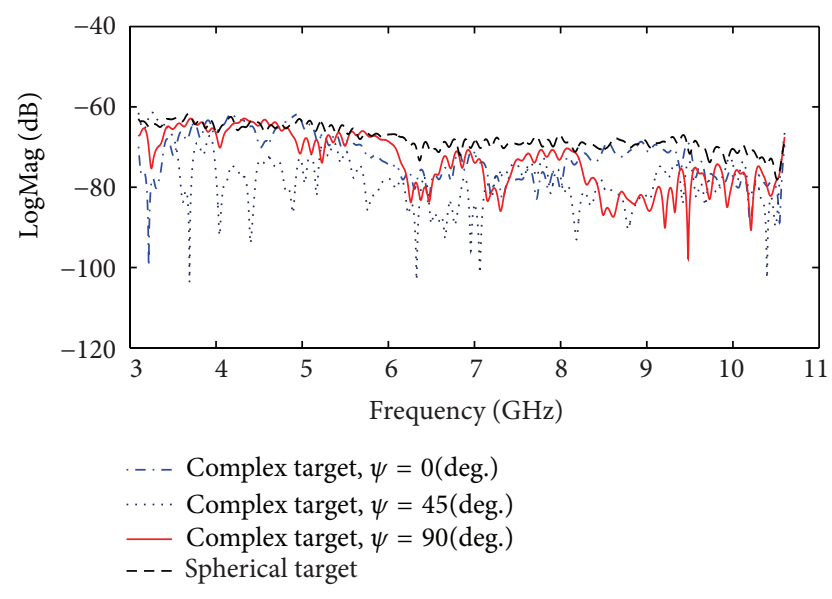

FIGURE 18: Measured frequency-domain data of the fabricated targets.

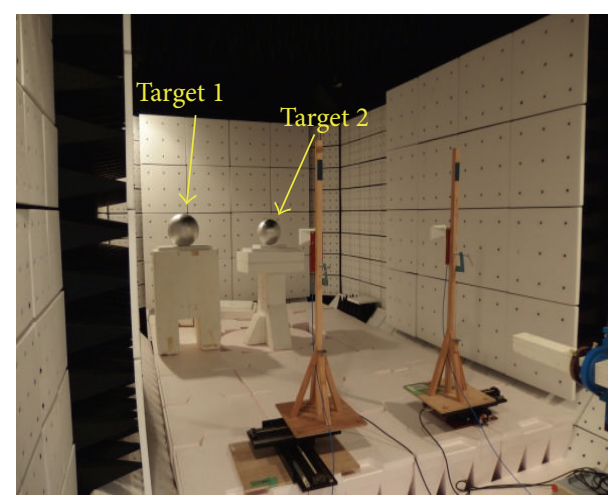

Figure 19: Measurement scenario in a radio anechoic chamber.

The estimation errors from measurement campaign were plotted in Figure 20. In the figure, the estimation errors were shown using signal bandwidths from 10 to $1000 \mathrm{MHz}$ and $f_{c}$ of 3.6, 5.0 and $8.4 \mathrm{GHz}$. As demonstrated in the numerical simulations, the measurement results also indicated a decreasing trend in estimation errors with increasing signal bandwidth, especially in the case of complex targets. The estimation errors of complex targets were worse than the sphere targets, where maximum of $7^{\circ}$ of estimation errors was observed. Both cases marked poorer performance compared to the simulations since the experimental measurement included plane wave modeling errors and other maneuvering factors. Furthermore, no significant difference in estimation performance was observed when choosing different center frequencies.

A series of experiments was conducted to evaluate the localization performance of the proposed scheme. A single complex target was positioned in several locations in the radio anechoic chamber. The positions of the target are summarized in Table 2. The target was localized using the standard triangulation method, adopting the proposed scheme. In addition, localization using DOA and time of
TABLE 2: Target positions in a radio anechoic chamber.

\begin{tabular}{lcc}
\hline $\begin{array}{l}\text { Target } \\
\text { positions }\end{array}$ & Actual DOD and DOA & $\begin{array}{c}\text { Target distance } \\
\text { from radar }\end{array}$ \\
\hline A & $(\phi, \theta)=\left(15^{\circ},-43^{\circ}\right)$ & $1.5 \mathrm{~m}$ \\
B & $(\phi, \theta)=\left(-3^{\circ},-24^{\circ}\right)$ & $2.2 \mathrm{~m}$ \\
C & $(\phi, \theta)=\left(-17^{\circ},-1^{\circ}\right)$ & $3.0 \mathrm{~m}$ \\
D & $(\phi, \theta)=\left(-21^{\circ}, 5^{\circ}\right)$ & $3.3 \mathrm{~m}$ \\
\hline
\end{tabular}

direction-of-arrival (TDOA) was also performed for comparison. The DOA-TDOA proposed method uses the DOA formulated from majority decisions of estimations at each subfrequency, and the TDOA was obtained from the time of arrival of the radar signal observed at Txl-Rxl antenna pair. The same MIMO array configuration as in the previous experiments was utilized, and the number of snapshots was 50. Considering the target location in an $x-y$ plane, the localization errors are defined as

$$
\Delta R=\sqrt{\left(\Delta x^{2}+\Delta y^{2}\right)}
$$

where $\Delta x$ and $\Delta y$ are the ranging errors along the $x$ and $y$ axes. The results of the measurements were plotted in Figure 21. In the case of triangulation, less than $1 \mathrm{~m}$ of error was obtained when using signal bandwidth of $1000 \mathrm{MHz}$. Using a $50 \mathrm{MHz}$ signal resulted in lower accuracy. The DOATDOA method also produced good results when using a $1000 \mathrm{MHz}$ signal and marked poor accuracy when using a $50 \mathrm{MHz}$ bandwidth, due to low range resolution of TDOA. Both localization methods showed slightly lower accuracy when the target is further away from the radar due to decrease in SNR.

\section{Concluding Remarks}

The performance of a joint DOD and DOA estimation in a UWB MIMO radar detecting fluctuating targets was evaluated through numerical simulations and experimental evaluations. From the investigation, it was found that in detecting targets with fluctuating RCS (in this case Weibull distributed RCS against frequency), it is essential to use large signal bandwidth to reduce the estimation error using the proposed algorithm. When taking wider signal bandwidth, the usage of majority decisions from the angle histograms resulted in good estimation performance compared to the conventional spectrum averaging method. We concluded based on the work that the proposed scheme was a suitable candidate to implement joint angle estimation in MIMO radar using ultra wideband signal. Although the resolution of target localization based on DOD and DOA estimations, in general, does not depend on the signal bandwidth, it was demonstrated that utilization of wider bandwidth in the proposed scheme leads to improvement of estimation performance, considering that the targets have fluctuating RCS in the frequency domain. Since the proposed scheme 


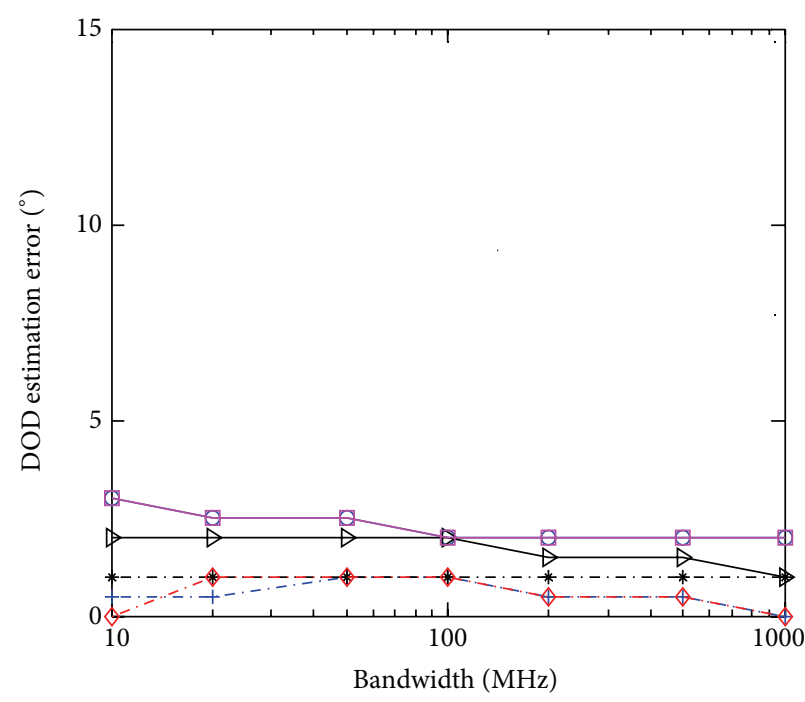

$\begin{array}{ll}\square \text { Target } 1, f_{c}=3.6 \mathrm{GHz} & --+- \text { Target } 2, f_{c}=3.6 \mathrm{GHz} \\ \square-\text { Target } 1, f_{c}=5 \mathrm{GHz} & -*-\text { Target } 2, f_{c}=5 \mathrm{GHz} \\ \square \quad \text { Target } 1, f_{c}=8.4 \mathrm{GHz} & -\downarrow-\text { Target } 2, f_{c}=8.4 \mathrm{GHz}\end{array}$

(a)

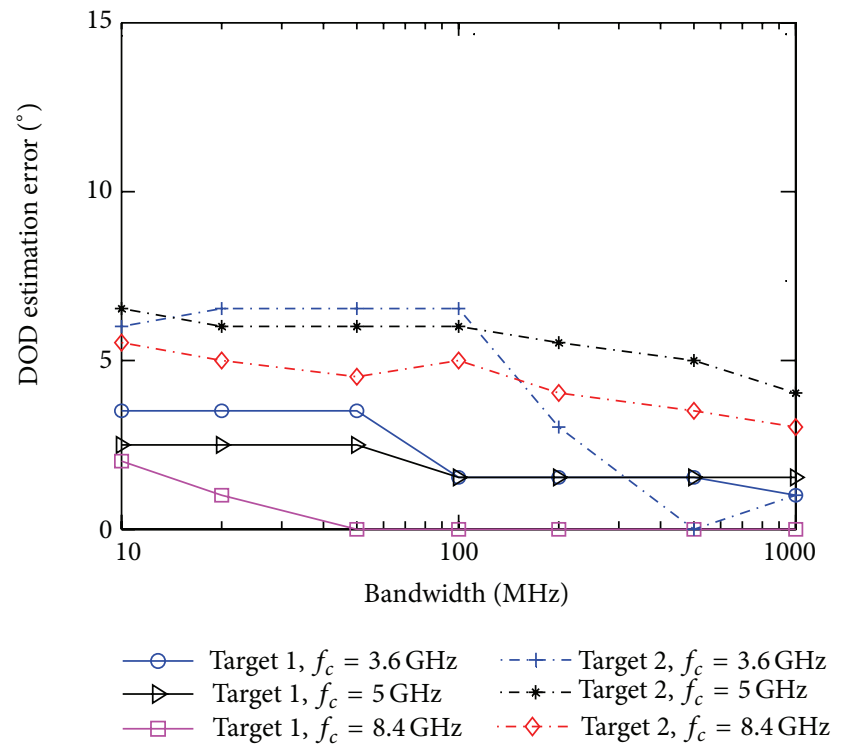

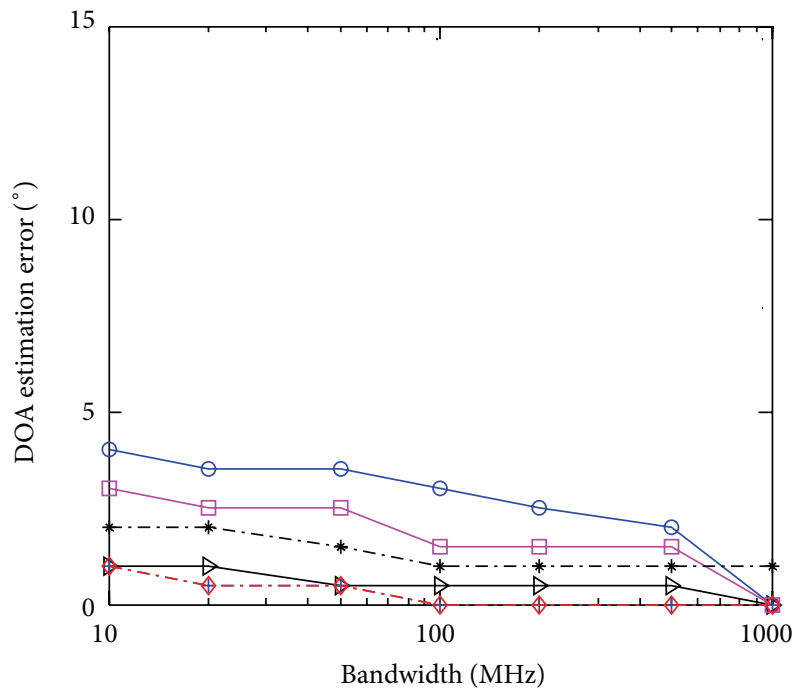

$\rightarrow$ Target $1, f_{c}=5 \mathrm{GHz} \quad-\quad *$ - Target $2, f_{c}=5 \mathrm{GHz}$ $\square$ Target $1, f_{c}=8.4 \mathrm{GHz} \quad-\diamond \cdot$ Target $2, f_{c}=8.4 \mathrm{GHz}$

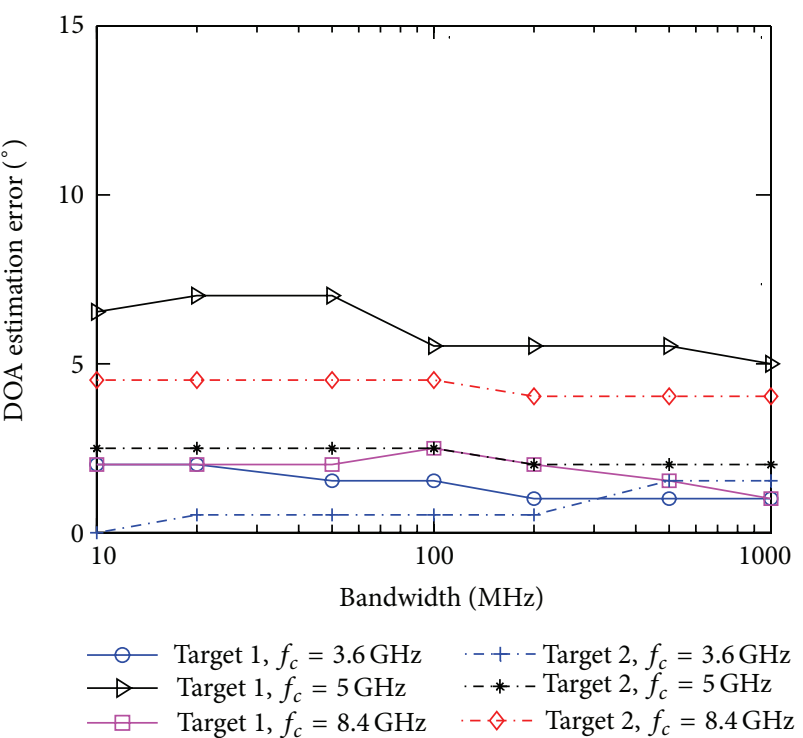

(b)

FIGURE 20: Estimation errors against bandwidth obtained from measurements: (a) spherical and (b) complex targets.

utilizes multiple subcarriers, it is suitable to be extended to an OFDM-based radar system.

\section{Appendix}

\section{MIMO Radar Virtual Array Using Nonuniform Array Configuration}

The MIMO antennas could be arranged in such a way that they produce a filled virtual array; however, the filled virtual array tends to consist of less number of unique virtual elements due to redundant elements. A few examples are depicted in Figure 22. Here, we illustrate the physical transmitting and receiving antennas position using the notation $\{1111\}$, where each entry corresponds to the number of antennas at the particular location on the $\lambda / 2$ grid, and $*$ is the convolutional operator. The results of the convolution of the transmitting and receiving antenna positions are shown on the right side of each figure. As depicted in Figure 22(a), the usage of uniform linear array on both transmitting and receiving arrays resulted in virtual arrays with significant number of redundant elements. 


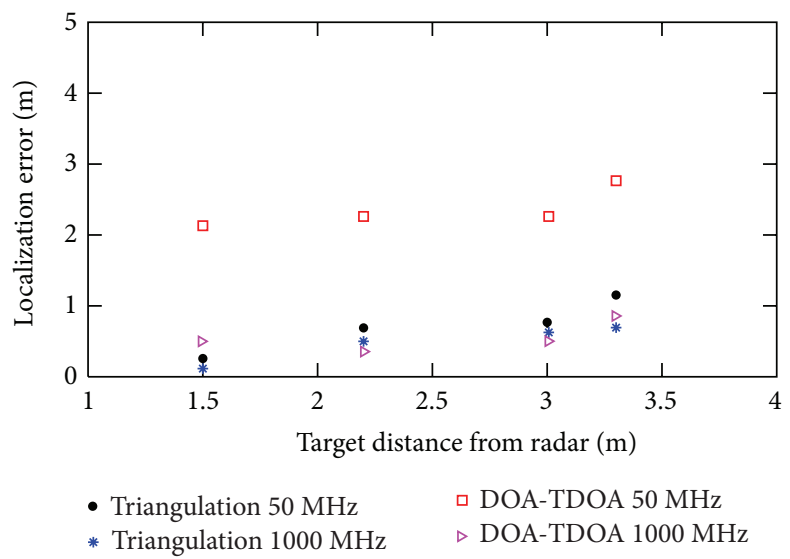

FIGURE 21: Localization errors from measurements in a radio anechoic chamber.

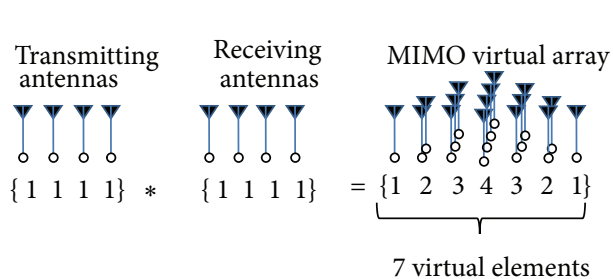

(a)

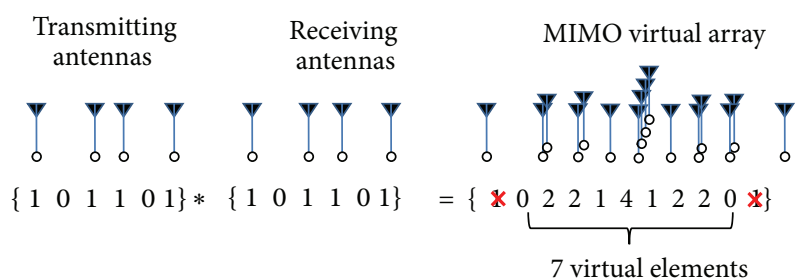

(b)

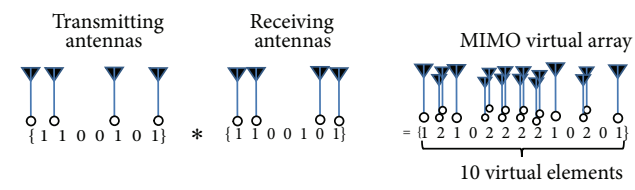

(c)

FIGURE 22: Illustration of examples of virtual arrays in MIMO radars: (a) uniform linear array, (b) truncated filled array, and (c) nonuniform array.

Configuration in Figure 22(b) produces a larger number of unique virtual elements. However, in order to obtain a filled array, truncation of the elements at the edge of the virtual array is required, thus reducing the array length. Meanwhile, the nonuniform MIMO array used in Figure 22(c) yielded a larger number of virtual antenna elements, since it has less number of redundant elements.

\section{Conflict of Interests}

The authors declare that there is no conflict of interests regarding the publication of this paper.

\section{References}

[1] E. Fishler, A. Haimovich, R. S. Blum, L. J. Cimini Jr., D. Chizhik, and R. A. Valenzuela, "Spatial diversity in radars-models and detection performance," IEEE Transactions on Signal Processing, vol. 54, no. 3, pp. 823-838, 2006.
[2] A. M. Haimovich, R. S. Blum, and L. J. Cimini, "MIMO radar with widely separated antennas," IEEE Signal Processing Magazine, vol. 25, no. 1, pp. 116-129, 2008.

[3] J. Li and P. Stoica, "MIMO radar with colocated antennas," IEEE Signal Processing Magazine, vol. 24, no. 5, pp. 106-114, 2007.

[4] X. H. Wu, A. A. Kishk, and A. W. Glisson, "MIMO-OFDM radar for direction estimation," IET Radar, Sonar \& Navigation, vol. 4, no. 1, pp. 28-36, 2010.

[5] X. Zhang, L. Xu, L. Xu, and D. Xu, "Direction of departure (DOD) and direction of arrival (DOA) estimation in MIMO radar with reduced-dimension mUSIC," IEEE Communications Letters, vol. 14, no. 12, pp. 1161-1163, 2010.

[6] B. Yao, W. Wang, and Q. Yin, "DOD and DOA estimation in bistatic non-uniform multiple-input multiple-output radar systems," IEEE Communications Letters, vol. 16, no. 11, pp. 17961799, 2012.

[7] G. Zheng, M. Yang, W. Guo, and B. Chen, "Joint DOD and DOA estimation for bistatic polarimetric MIMO radar," in Proceedings of the IEEE 11th International Conference on Signal Processing (ICSP '12), pp. 329-332, Beijing, China, October 2012.

[8] F. Liu and J. Wang, "AD-MUSIC for jointly DOA and DOD estimation in bistatic MIMO radar system," in Proceedings of the 
International Conference on Computer Design and Applications (ICCDA '10), pp. V4455-V4458, June 2010.

[9] M. A. Haleem, A. Haimovich, and R. S. Blum, "Sidelobe mitigation in MIMO radar with multiple subcarriers," in Proceedings of the IEEE Radar Conference (RADAR '09), pp. 1-6, May 2009.

[10] X. H. Wu, A. A. Kishk, and A. W. Glisson, "MIMO-OFDM radar for direction estimation," IET Radar, Sonar and Navigation, vol. 4, no. 1, pp. 28-36, 2010.

[11] X. Zhuge, A. G. Yarovoy, T. Savelyev, and L. Ligthart, "Modified kirchhoff migration for UWB MIMO array-based radar imaging," IEEE Transactions on Geoscience and Remote Sensing, vol. 48, no. 6, pp. 2692-2703, 2010.

[12] X. Zhuge and A. G. Yarovoy, "Study on two-dimensional sparse MIMO UWB arrays for high resolution near-field imaging," IEEE Transactions on Antennas and Propagation, vol. 60, no. 9, pp. 4173-4182, 2012.

[13] I. Pasya, N. Iwakiri, and T. Kobayashi, "Joint direction-ofdeparture and direction-of-arrival estimation in an ultrawideband MIMO radar system," in Proceedings of the IEEE Radio Wireless Symposium (RWS '14), pp. 52-54, Newport Beach, Calif, USA, 2014.

[14] P. de Silva and C. K. Seow, "Performance of MIMO RADAR using two-way music," in Proceedings of the Progress in Electromagnetics Research Symposium (PIERS '13), pp. 84-87, March 2013.

[15] N. Iwakiri and T. Kobayashi, "Ultra-wideband time-of-arrival and angle-of-arrival estimation using a signal model based on measurements," IEICE Transactions on Fundamentals of Electronics, Communications and Computer Sciences, vol. 90, no. 11, pp. 2345-2353, 2007.

[16] W. Zhang, W. Liu, J. Wang, and S. Wu, "Joint transmission and reception diversity smoothing for direction finding of coherent targets in MIMO radar," IEEE Journal of Selected Topics in Signal Process, vol. 8, no. 1, pp. 115-124, 2014.

[17] D. Wilcox, M. Sellathurai, and T. Ratnarajah, "Subspace methods and spatial diversity in radars," in Proceedings of the IEEE Radar Conference (RADAR '08), pp. 1-5, Rome, Italy, May 2008.

[18] Y. Chen, Y. Nijsure, C. Yuen, Y. H. Chew, Z. Ding, and S. Boussakta, "Adaptive distributed MIMO radar waveform optimization based on mutual information," IEEE Transactions on Aerospace and Electronic Systems, vol. 49, no. 2, pp. 1374-1385, 2013.

[19] V. Mangulis, "Frequency diversity in low-angle radar tracking," IEEE Transactions on Aerospace and Electronic Systems, vol. 17, no. 1, pp. 149-153, 1981.

[20] J. J. Zhang and A. Papandreou-Suppappola, "MIMO radar with frequency diversity," in Proceeedings of the International Waveform Diversity and Design Conference (WDD '09), pp. 208212, February 2009.

[21] M. Fujimoto, S. Ohaka, and T. Hori, "DOA estimation without antenna characteristics calibration for UWB signal by using sub-band processing," in Proceedings of the IEEE International Conference on Wireless Information Technology and Systems (ICWITS '10), pp. 1-4, September 2010.

[22] W.-Q. Wang, "Virtual antenna array analysis for MIMO synthetic aperture radars," International Journal of Antennas and Propagation, vol. 2012, Article ID 587276, 10 pages, 2012.

[23] L. Jian and P. Stoica, Eds., MIMO Radar Signal Processing, Wiley, New Jersey, NJ, USA, 2009.

[24] G. H. Golub and C. F. van Loan, Matrix Computations, Johns Hopkins University Press, Baltimore, Md, USA, 3rd edition, 1996.
[25] W. Buller, B. Wilson, L. Van Nieuwstadt, and J. Ebling, "Statistical modelling of measured automotive radar reflections," in Proceedings of the IEEE International Instrumentation and Measurement Technology Conference (I2MTC '13), pp. 349-352, May 2013.

[26] H. W. Chen, D. Yang, H. Q. Wang, X. Li, and Z. W. Zhuang, "Direction Finding for bistatic MIMO radar using EM maximum likelihood algorithm," Progress in Electromagnetics Research, vol. 141, pp. 99-116, 2013.

[27] H. L. van Trees, Optimum Array Processing, Wiley, New York, NY, USA, 2009. 

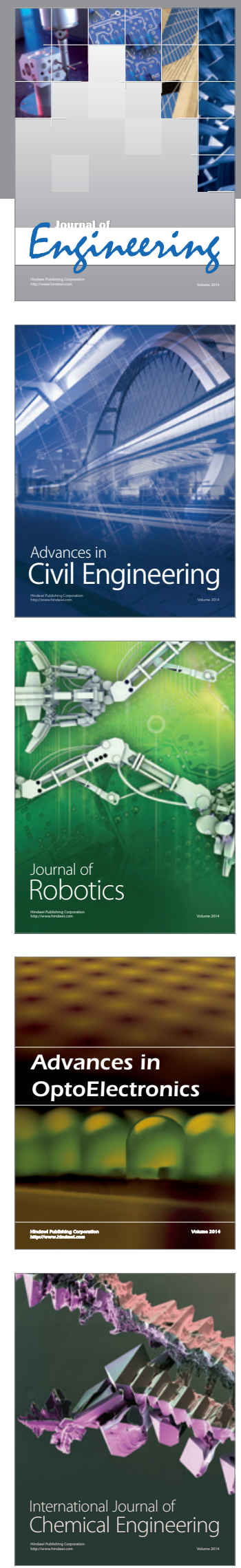

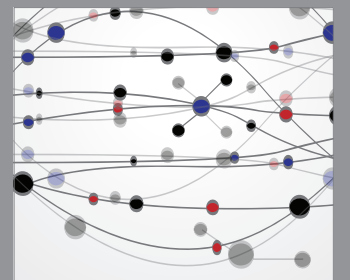

The Scientific World Journal
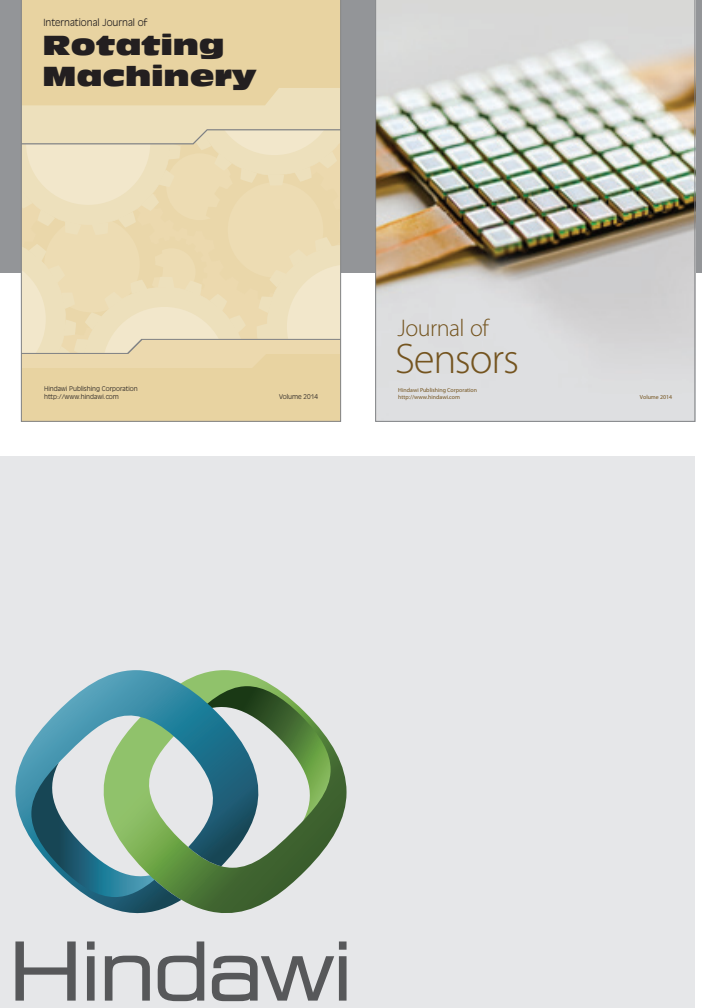

Submit your manuscripts at http://www.hindawi.com
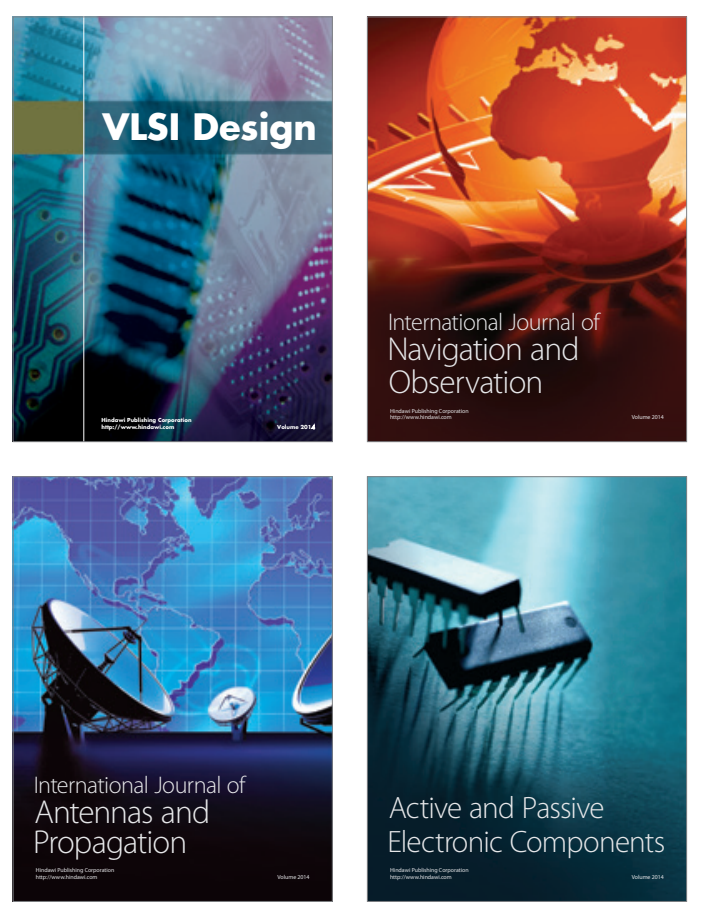
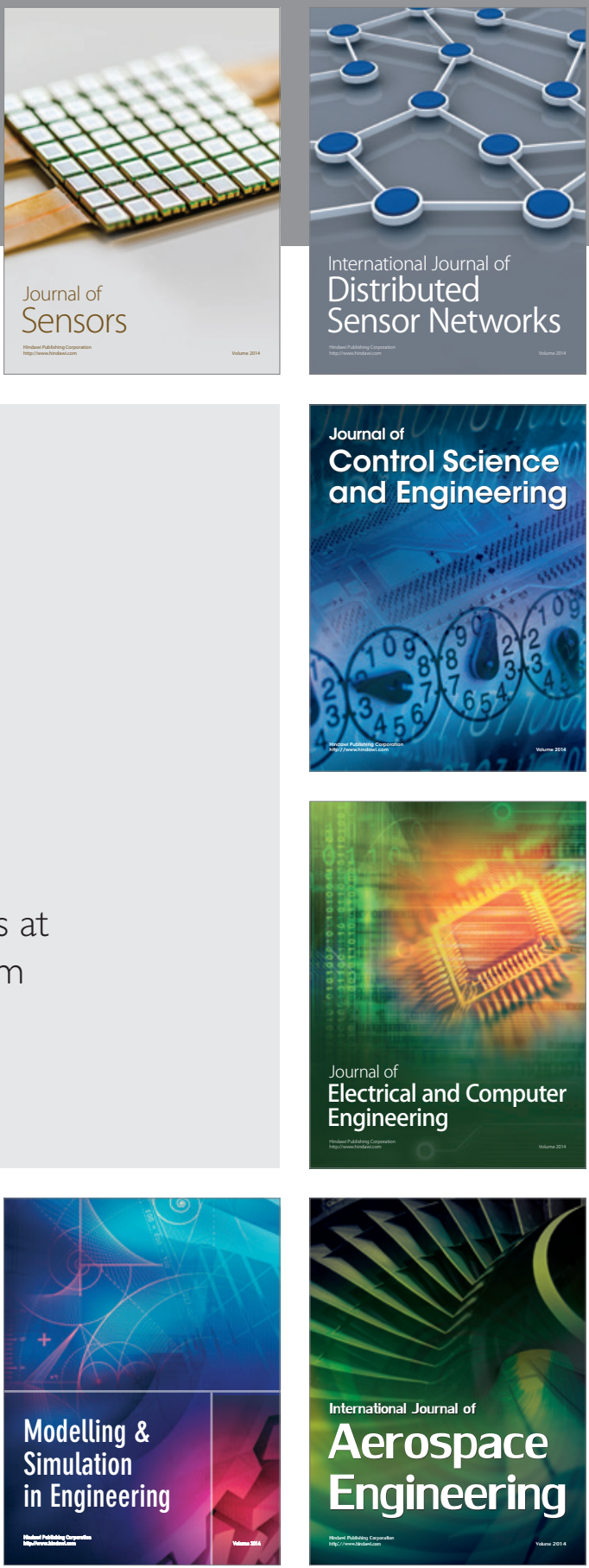

Journal of

Control Science

and Engineering
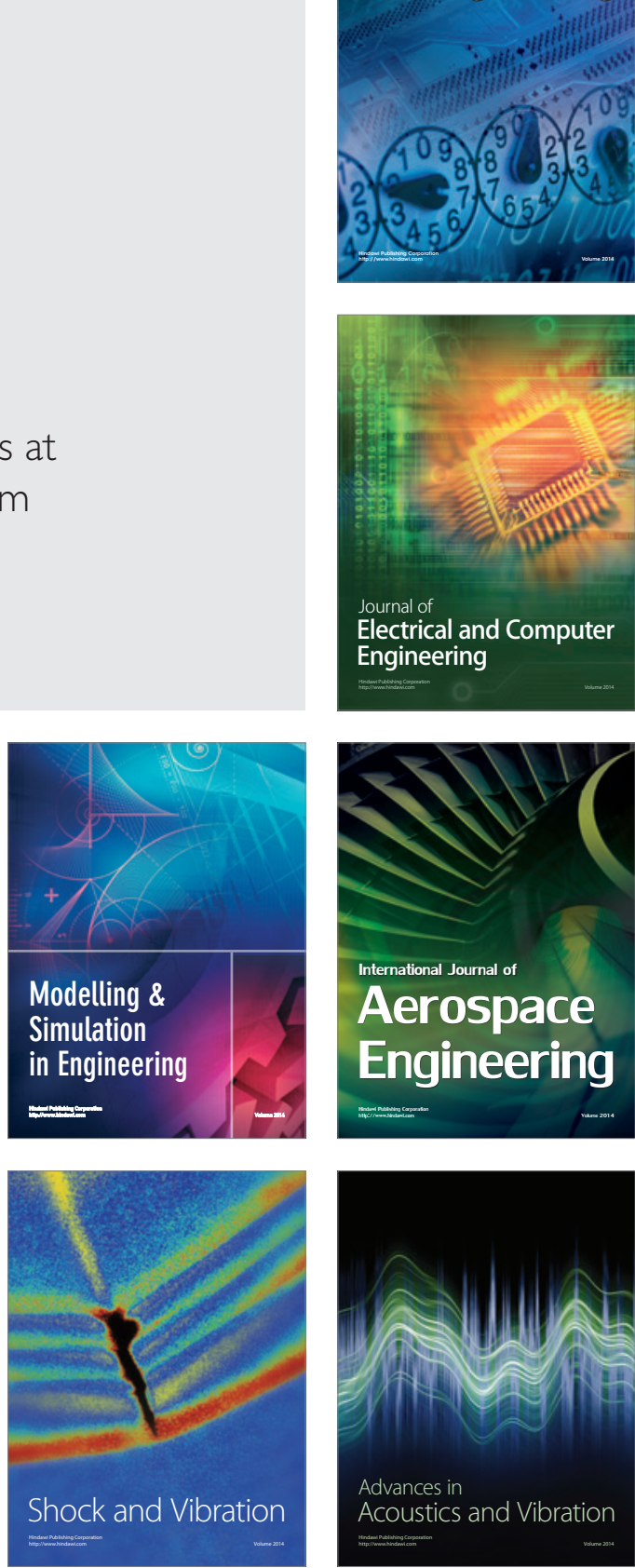